Open Access

\title{
Full-duplex distributed switch-and-stay networks with wireless energy harvesting: design and outage analysis
}

\author{
Quang Nhat Le', Nhu Tri Do' ${ }^{1}$ Vo Nguyen Quoc Bao² and Beongku An $3^{*}$ (D)
}

\begin{abstract}
Distributed switch and stay combining (DSSC) has been considered as an effective technique to achieve spatial diversity in a distributed manner with low processing complexity. In this paper, we incorporate DSSC into a full-duplex dual hop relaying system, where two energy-constrained relay nodes assist the information transmission from the source to the destination using energy wirelessly harvested from the source. By applying DSSC at the destination, this efficient technique not only improves performance of full-duplex relaying systems and increases the system diversity but also reduces the implementation and hardware complexity at the destination. We obtain tight approximate expression for outage probability in the case of using decode-and-forward (DF) relaying protocol and analytical expression for outage probability in the case of using amplify-and-forward (AF) relaying protocol. Numerical results show that our DSSC full-duplex relaying with wireless information and power transfer (WIPT) system achieves the full spatial diversity and has a better performance in terms of outage probability than that of the DSSC half-duplex relaying and conventional full-duplex relaying with WIPT system.
\end{abstract}

Keywords: Dual-hop systems, Full-duplex relaying, Distributed switch and stay combining (DSSC), Wireless information and power transfer (WIPT), Outage probability, Energy harvesting

\section{Introduction}

Wireless energy transfer through radio frequency (RF) signals has emerged as the promising and effective solution to supply power and prolong the lifetime of energy-constrained wireless networks. Since RF signals can carry both information and energy, it has recently attracted a lot of attention on research activities, especially on investigating simultaneously wireless information and power transfer (SWIPT) [1-3]. In [4], an energy constrained relay node using amplify-andforward (AF) protocol was considered based on the time switching and power splitting receiver architectures, respectively. Specifically, the authors investigated both the delay-limited and the non-delay-limited transmission

*Correspondence: beongku@hongik.ac.kr

'Department of Electronics and Computer Engineering, Hongik University, Sejong 30016, Korea

Full list of author information is available at the end of the article and derived the analytical expressions for the outage probability and ergodic capacity, respectively. Later on, the same authors extended the analysis to the throughput and ergodic capacity of DF relaying systems in [5]. Based on the time switching receiver architecture, the authors in [6] proposed adaptive time switching protocols for RF energy harvesting and information transmission for both AF and DF networks. They obtained analytical expressions of the achievable throughput for both the continuous and the discrete time switching protocols. It is pointing out that these authors only consider half-duplex relaying mechanism, where relay node cannot receive and transmit data simultaneously in the same frequency band.

With the advance of antenna and signal processing technology, full-duplex (FD) has received significant attention, where wireless nodes can receive and transmit information simultaneously in the same frequency band (see references [7-10] and therein). In 
[11], the authors investigated harvest-use (HU) relaying system with half-duplex and full-duplex operation mode. The main conclusion in [11] is that FD seems to be an attractive and promising technology for HUbased cooperative networks. In [12], the authors considered a time switching-based full-duplex wireless powered relaying system, where the relay node operated in fullduplex mode with simultaneously information reception and information transmission. Specifically, they provided an analytical characterization of the achievable throughput of three different communication modes, namely, instantaneous transmission, delay-constrained transmission, and delay tolerant transmission by optimizing time splitting ratio. In [13], the authors examined a beamforming scheme in a time switching-based relaying energy harvesting system with multi-antenna full-duplex DF relays. Moreover, a full-duplex wireless-powered transmission system with simultaneous information reception and energy transmission was investigated in [14]. In [15], the authors proposed a novel two-phase protocol for an AF relay to activate full-duplex transmission. Especially, they regarded the energy from the loopback-interference channel as useful energy and integrated this energy into the wireless-powered transfer process, and the optimal power allocation and beamforming design at the relay node were derived. However, the performance and diversity of full-duplex relaying with WIPT networks can be further improved.

There is a lot of research interest in the diversity community for diversity schemes and performance analyses have recently appeared $[16,17]$. Motivated by this, in this paper, we utilize distributed switch-and-stay combining (DSSC) technique at the destination for the case where two relaying terminals are used to improve the performance of full-duplex relaying with WIPT networks and to reduce the implementation complexity at the destination. Our system may be the case in practical scenarios where the destination may not support complex functions, e.g., employing a diversity combiner due to a tiny hardware constraint wireless node which has limited battery power and signal processing capability or the direct link between the source and destination does not exist due to deep shadowing [18-20,22]. The term "swich-and-stay combining " is the efficient diversity-exploiting technique that switches between signals from two branches based on their instantaneous received signal-to-noise ratio (SNR). SSC has received significant attention, and there have been a lot of research works which investigate the performance of this technique. In [21] and [22], the authors proposed DSSC technique applied to one and two relays with DF. The performance of such systems was derived in terms of outage and error probability over Rayleigh fading channel. In [23], the end-to-end error performance of Binary Phase Shift Keying (BPSK)-modulated DSSC scheme with a single antenna fixed-gain AF relay was examined under Rayleigh fading conditions. The combination of distributed SSC and relay selection had been proposed and investigated in terms of bit error rate in [24]. In [25], the performance of distributed SSC and relay selection in terms of outage probability was considered. In [26], the authors examined DSSC and relay selection with the combination of signal space diversity to further improve diversity and error performance gains. Throughout these papers, it is pointing out that DSSC has its ability to improve system performance and the destination do not need to use a combiner, which significantly reduces the complexity level at the destination. The main contributions of our paper are summerized as follows.

- We proposed the application of distributed switch and stay combining (DSSC) to full-duplex relaying with WIPT networks to improve system performance. This is the first work of considering this combination.

- We derived the performance of our proposed system in terms of the outage probability (OP) over Rayleigh fading channels for both two typical relaying protocols, AF and DF protocols. Through some simulation results, it is shown that our proposed protocol can attain the full spatial diversity if the preselected switching threshold is chosen equal or higher than the outage threshold. Otherwise, the diversity of one is obtained. This is verified by numerical results and diversity analysis.

- We showed that our proposed system has the better performance than the DSSC half-duplex relaying system and conventional full-duplex relaying one according to the system outage probability obtained by numerical results.

The rest of the paper is organized as follows. In Section 2, DSSC full-duplex relaying with WIPT systems is presented. In Section 3, we formulate the outage probability of our proposed system in two different cases: two relays operate in DF mode and two relays operate in AF mode. Section 4 provides diversity analysis of DF and AF cases. In Section 5, we consider half-duplex relaying using DSSC which serves as a benchmark. In Section 6, numerical results are presented. Finally, Section 7 concludes the paper.

\section{System model}

\subsection{System description}

We consider a dual hop full-duplex relaying system, which is illustrated in Fig. 1, consisting of one source $S$, one destination $\mathrm{D}$, and two relays $R_{1}$ and $R_{2}$. We assume that 


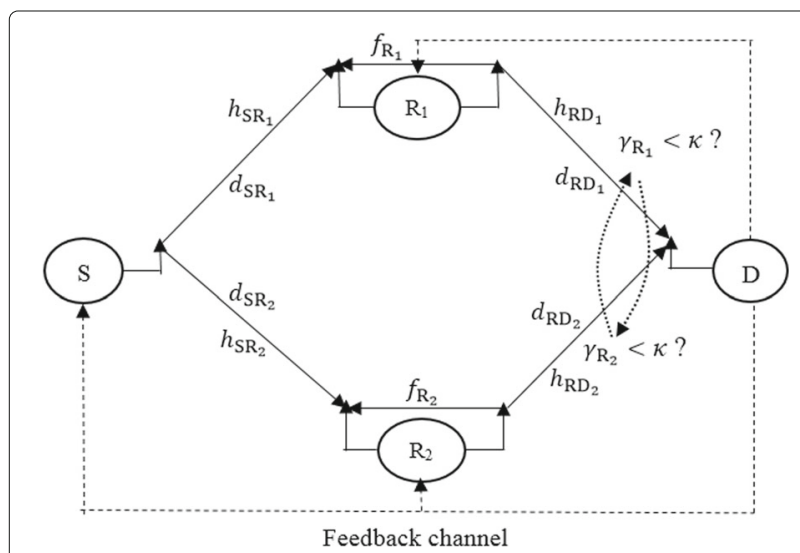

Fig. 1 System model, where $S, R_{1}, R_{2}$, and $D$ denote the source, relay 1 , relay 2 , and the destination, respectively

the direct transmission link between the source and the destination is not available and communication from the source to the destination is only conducted through the help of two relays. The source and the destination are equipped with single antenna and each relay is equipped with two antennas, i.e., one for information transmission and one for information reception. The source and the destination have their own energy, while two relays only have limited power supply, and rely on external charging through harvesting energy from the source. The model is practical since the relay only harvests energy to help the source transmission and uses its own energy for its own data transmission.

All wireless links exhibit fading and addictive white Gaussian noise (AWGN) [11]. The channels are modelled to be frequency-flat, independent, and identically distributed Rayleigh block fading. This means that the channel coefficients are constant over a single block time $T$, but vary independently from block to block. Let $h_{\mathrm{SR}_{1}}, h_{\mathrm{R}_{1} \mathrm{D}}, h_{\mathrm{SR}_{2}}, h_{\mathrm{R}_{2} \mathrm{D}}, f_{\mathrm{R}_{1}}$, and $f_{\mathrm{R}_{2}}$ be the channel coefficients from the source to relay $R_{1}$, relay $R_{1}$ to the destination, the source to relay $R_{2}$, relay $R_{2}$ to the destination, the loopback interference channel of relay $R_{1}$ and the loopback interference channel of relay $R_{2}$, respectively. Due to Rayleigh fading, the channel powers, denoted by $\left|h_{\mathrm{SR}_{1}}\right|^{2},\left|h_{\mathrm{R}_{1} \mathrm{D}}\right|^{2},\left|h_{\mathrm{SR}_{2}}\right|^{2},\left|h_{\mathrm{R}_{2} \mathrm{D}}\right|^{2},\left|f_{\mathrm{R}_{1}}\right|^{2}$, and $\left|f_{\mathrm{R}_{2}}\right|^{2}$, are independent and exponential random variables, whose means are $\lambda_{h_{\mathrm{SR}_{1}}}, \lambda_{h_{\mathrm{R}_{1} \mathrm{D}}}, \lambda_{h_{\mathrm{SR}_{2}}}, \lambda_{h_{R_{2} \mathrm{D}}}, \lambda_{f_{R_{1}}}$, and $\lambda_{f_{\mathrm{R}_{2}}}$, respectively. Further, the SNR of two branches $\mathrm{S} \rightarrow \mathrm{R}_{1} \rightarrow \mathrm{D}$ and $S \rightarrow R_{2} \rightarrow$ D are represented by $\gamma_{R_{1}}$ and $\gamma_{R_{2}}$, respectively.

In this paper, information is transmitted between the source and the destination through either $R_{1}\left(S \rightarrow R_{1} \rightarrow D\right)$ or $\mathrm{R}_{2}\left(\mathrm{~S} \rightarrow \mathrm{R}_{2} \rightarrow \mathrm{D}\right)$ link. Therefore, some technique need to be applied at the destination to combine two signals from two links or select one of the twos from two links. To increase the system diversity and also to reduce the complexity at the destination, we propose distributed switch and stay combining (DSSC) technique. The operation of this technique in our model is described as follows. In each transmission block time $T$, only one link (either $\mathrm{S} \rightarrow \mathrm{R}_{1} \rightarrow \mathrm{D}$ or $\left.\mathrm{S} \rightarrow \mathrm{R}_{2} \rightarrow \mathrm{D}\right)$ is active. To determine the active link, depending on which link is active in the current block time $T$, the destination node compares the received SNR (which equals to $\gamma_{R_{1}}$ or $\gamma_{R_{2}}$ ) with the given threshold $\kappa$. The switching occurs (the alternative link will be used in the next block time $T$ ) when the instantaneous SNR of the currently selected link falls below the threshold $\kappa$ regardless of the current instantaneous SNR of the alternative link. The switching process will be implemented by an appropriate feedback signal sent from destination to the source and relays through the dedicated feedback channel, indicating a switching on the transmission path. We assume that there exists an error-free feedback channel between the source and the destination. More specifically, the source signal reaches the destination through either $\mathrm{S} \rightarrow \mathrm{R}_{1} \rightarrow \mathrm{D}$ link or $\mathrm{S} \rightarrow \mathrm{R}_{2} \rightarrow \mathrm{D}$ link and in each transmission block time $T$, only one link is active.

To activate full-duplex transmission at two relays, we use the two-phase protocol in [15], which is described in Fig. 2. In the first phase of duration $T / 2$, the source transmits information to relay $R_{1}$ or $R_{2}$. In the second phase of duration $T / 2$, depending on the AF or DF protocol which is used at the selected relay, the selected relay processes information and then forwards this information to the destination, and concurrently, the selected relay also receives dedicated energy signal from the source for energy harvesting. Therefore, full-duplex transmission is conducted in the second phase, which the selected relay simultaneously harvests energy and transmits information to the destination. Besides that, the loopback signal created from the transmitting antenna at the selected relay, which is regarded as loopback interference, thus requires additional energy to apply interference cancellation methods to mitigate this problem. As same as in [15], we also consider that self-interference link as beneficial link because the loopback energy can be recycled by the relay

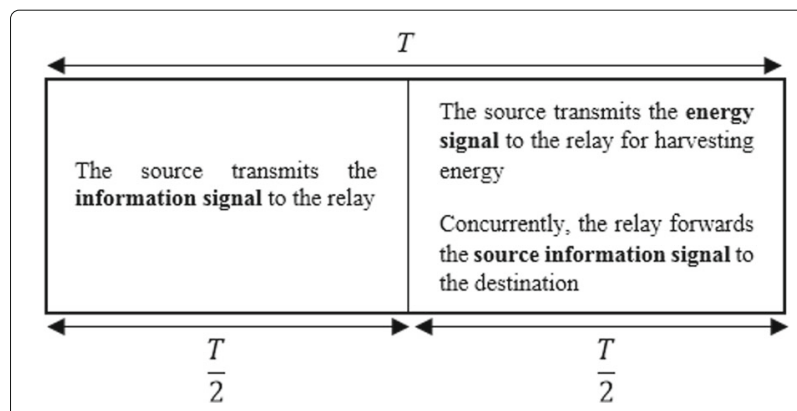

Fig. 2 Block time $T$ for full-duplex dual hop relaying systems 
and the relay harvests and uses this energy for information transmission in addition to harvested energy from the source signal. For simplicity, in this paper, we assume that there are no interference signals from the transmitting antenna of relay $R_{1} / R_{2}$ to the receiving antenna of relay $R_{2} / R_{1}$ since only one relay is active when using DSSC technique, so we do not need to consider the link between two relays [18-20,22]. We further assume that the relay of the inactive link will keep idle, i.e., no energy is harvested.

\subsection{Signal modeling}

In this section, we analyze signal modeling in two phases. In each phase, we consider two cases: relay $R_{1}$ is used for information transmission ( $\rightarrow R_{1} \rightarrow D$ is active) and relay $R_{2}$ is used for information transmission $\left(S \rightarrow R_{2} \rightarrow D\right.$ is active).

\subsubsection{In the first phase}

In case of using relay $R_{1}$, the received signal at relay $R_{1}$ can be expressed as

$$
y_{\mathrm{SR}_{1}}=\frac{h_{\mathrm{SR}_{1}}}{\sqrt{d_{\mathrm{SR}_{1}}^{m}}} x_{\mathrm{S}}+n_{\mathrm{R}_{1}},
$$

where $d_{\mathrm{SR}_{1}}$ is the distance between the source and relay $\mathrm{R}_{1}, m$ is the path loss exponent, $x_{\mathrm{S}}$ is the information symbol with $E\left\{\left|x_{S}\right|^{2}\right\}=\mathcal{P}_{S}, E\{$.$\} denotes the expectation$ operation, $\mathcal{P}_{\mathrm{S}}$ is the source transmit power and $n_{\mathrm{R}_{1}}$ is the zero mean additive white Gaussian noise (AWGN) with variance $N_{0}$.

Hence, the instantaneous $S N R$ of $S-R_{1}$ link is given by

$$
\gamma_{\mathrm{SR}_{1}}=\frac{\left|h_{\mathrm{SR}_{1}}\right|^{2} \mathcal{P}_{\mathrm{S}}}{N_{0} d_{\mathrm{SR}_{1}}^{m}}=\frac{\left|h_{\mathrm{SR}_{1}}\right|^{2} \bar{\gamma}}{d_{\mathrm{SR}_{1}}^{m}}
$$

where $\bar{\gamma}=\frac{\mathcal{P}_{\mathrm{S}}}{N_{0}}$ is the average transmit SNR.

In case of using relay $R_{2}$, the received signal at relay $R_{2}$ can be expressed as

$$
y_{\mathrm{SR}_{2}}=\frac{\left|h_{\mathrm{SR}_{2}}\right|^{2}}{\sqrt{d_{\mathrm{SR}_{2}}^{m}}} x_{\mathrm{S}}+n_{\mathrm{R}_{2}},
$$

Hence, the instantaneous SNR of $S-R_{2}$ link is given by

$$
\gamma_{\mathrm{SR}_{2}}=\frac{\left|h_{\mathrm{SR}_{2}}\right|^{2} \mathcal{P}_{\mathrm{S}}}{N_{0} d_{\mathrm{SR}_{2}}^{m}}=\frac{\left|h_{\mathrm{SR}_{2}}\right|^{2} \bar{\gamma}}{d_{\mathrm{SR}_{2}}^{m}} .
$$

\subsubsection{In the second phase}

In this paper, we consider both AF and DF relaying protocols.
In case of using relay $R_{1}$, with the AF protocol, the relay $R_{1}$ amplifies the input signal by a factor $\beta_{R_{1}}$, which is given by [27]

$$
\beta_{\mathrm{R}_{1}}^{2}=\frac{\mathcal{P}_{\mathrm{R}_{1}}}{\left|h_{\mathrm{SR}_{1}}\right|^{2} \mathcal{P}_{\mathrm{S}} / d_{\mathrm{SR}_{1}}^{m}+N_{0}}
$$

where $\mathcal{P}_{R_{1}}$ is the transmit power of relay $R_{1}$.

Hence, the transmit signal of relay $R_{1}$ is given by [27]

$$
x_{\mathrm{R}_{1}}=\beta_{\mathrm{R}_{1}} y_{\mathrm{SR}_{1}} .
$$

With the DF protocol, the relay first decodes the original signal from the source and then forwards the re-encoded version to the destination. Hence, the transmit signal of relay $R_{1}$ can be expressed as [27]

$$
x_{\mathrm{R}_{1}}=\sqrt{\frac{\mathcal{P}_{\mathrm{R}_{1}}}{\mathcal{P}_{\mathrm{S}}}} x_{\mathrm{S}} .
$$

The received signal at the destination can be expressed as

$$
y_{D_{\mathrm{R}_{1}}}=\frac{h_{\mathrm{R}_{1} \mathrm{D}}}{\sqrt{d_{\mathrm{R}_{1} \mathrm{D}}^{m}}} x_{\mathrm{R}_{1}}+n_{\mathrm{D}},
$$

where $x_{R_{1}}$ is the transmit signal of relay $R_{1}$ with $E\left\{\left|x_{R_{1}}\right|^{2}\right\}$ $=\mathcal{P}_{R_{1}}, \mathcal{P}_{R_{1}}$ is the transmit power of relay $R_{1}, d_{R_{1} D}$ is the distance between relay $R_{1}$ and the destination and $n_{D}$ is the zero mean AWGN with variance $N_{0}$.

Hence, the instantaneous $S N R$ of $R_{1}-D$ link is given by

$$
\gamma_{\mathrm{R}_{1} \mathrm{D}}=\frac{\left|h_{\mathrm{R}_{1} \mathrm{D}}\right|^{2} \mathcal{P}_{\mathrm{R}_{1}}}{N_{0} d_{\mathrm{R}_{1} \mathrm{D}}^{m}}
$$

In case of using relay $R_{2}$, with the AF protocol, the relay $R_{2}$ amplifies the input signal by a factor $\beta_{R_{2}}$ which is given by [27]

$$
\beta_{\mathrm{R}_{2}}^{2}=\frac{\mathcal{P}_{\mathrm{R}_{2}}}{\left|h_{\mathrm{SR}_{2}}\right|^{2} \mathcal{P}_{\mathrm{S}} / d_{\mathrm{SR}_{2}}^{m}+N_{0}},
$$

where $\mathcal{P}_{R_{2}}$ is the transmit power of relay $R_{2}$.

Hence, the transmit signal of relay $R_{2}$ is given by [27]

$$
x_{\mathrm{R}_{2}}=\beta_{\mathrm{R}_{2}} y_{\mathrm{SR}_{2}} .
$$

With the DF protocol, the transmit signal of relay $R_{2}$ can be expressed as [27]

$$
x_{\mathrm{R}_{2}}=\sqrt{\frac{\mathcal{P}_{\mathrm{R}_{2}}}{\mathcal{P}_{\mathrm{S}}} x_{\mathrm{S}}} .
$$


The received signal at destination can be expressed as

$$
y_{D_{\mathrm{R}_{2}}}=\frac{h_{\mathrm{R}_{2} \mathrm{D}}}{\sqrt{d_{\mathrm{R}_{2} \mathrm{D}}^{m}}} x_{\mathrm{R}_{2}}+n_{\mathrm{D}},
$$

Hence, the instantaneous SNR of $R_{2}-D$ link is given by

$$
\gamma_{\mathrm{R}_{2} \mathrm{D}}=\frac{\left|h_{\mathrm{R}_{2} \mathrm{D}}\right|^{2} \mathcal{P}_{\mathrm{R}_{2}}}{N_{0} d_{\mathrm{R}_{2} \mathrm{D}}^{m}}
$$

Concurrently with information transmission by active relay in the second phase, the relay harvests energy from the energy signal which is transmitted from the source. Here, we assume that the relay harvests dedicated energy from the source, but the relay also recycles its own energy from loopback self-interference channel [15].

In case of using relay $R_{1}$, the received signal by the receiving antenna of relay $R_{1}$ is expressed as

$$
y_{\mathrm{R}_{1}}=\frac{h_{\mathrm{SR}_{1}}}{\sqrt{d_{\mathrm{SR}_{1}}^{m}}} x_{\mathrm{E}}+f_{\mathrm{R}_{1}} x_{\mathrm{R}_{1}}+n_{\mathrm{R}_{1}},
$$

where $x_{\mathrm{E}}$ is the energy symbol from the source.

The transmit power at relay $R_{1}$ is given by [15]

$$
\begin{aligned}
\mathcal{P}_{\mathrm{R}_{1}} & =\frac{(T / 2) \eta\left(\left|h_{\mathrm{SR}_{1}}\right|^{2} \mathcal{P}_{\mathrm{S}}+\left|f_{\mathrm{R}_{1}}\right|^{2} \mathcal{P}_{\mathrm{R}_{1}}\right)}{d_{\mathrm{SR}_{1}}^{m}(T / 2)} \\
& =\frac{\eta\left|h_{\mathrm{SR}_{1}}\right|^{2} \mathcal{P}_{\mathrm{S}}}{d_{\mathrm{SR}_{1}}^{m}-\eta\left|f_{\mathrm{R}_{1}}\right|^{2}}
\end{aligned}
$$

where $\eta$ is a constant and denotes the energy conversion efficiency. Since we consider the self-interference link as useful link, thus from (16), the selected relay not only harvests energy from the source but it also scavenges energy from the self-interference link. Note that, we take into consideration particular case that the average energy used for transmission by the selected relay is equal to that being harvested [15]. Further, we ignore the negligible energy harvested from the receiver noise since the energy harvested from the source is much larger than that of the receiver noise [12].

In case of using relay $R_{2}$, the received signal by the receiving antenna of relay $R_{2}$ is expressed as

$$
y_{\mathrm{R}_{2}}=\frac{h_{\mathrm{SR}_{2}}}{\sqrt{d_{\mathrm{SR}_{2}}^{m}}} x_{\mathrm{E}}+f_{\mathrm{R}_{2}} x_{\mathrm{R}_{2}}+n_{\mathrm{R}_{2}},
$$

The transmit power at relay $R_{2}$ is given by

$$
\begin{aligned}
\mathcal{P}_{\mathrm{R}_{2}} & =\frac{(T / 2) \eta\left(\left|h_{\mathrm{SR}_{2}}\right|^{2} \mathcal{P}_{\mathrm{S}}+\left|f_{\mathrm{R}_{2}}\right|^{2} \mathcal{P}_{\mathrm{R}_{2}}\right)}{d_{\mathrm{SR}_{2}}^{m}(T / 2)} \\
& =\frac{\eta\left|h_{\mathrm{SR}_{2}}\right|^{2} \mathcal{P}_{\mathrm{S}}}{d_{\mathrm{SR}_{2}}-\eta\left|f_{\mathrm{R}_{2}}\right|^{2}} .
\end{aligned}
$$

\subsection{Mode of operation}

At the beginning of each transmission block time $T$, one link needs to be activated. Specifically, suppose that the $\mathrm{S} \rightarrow \mathrm{R}_{1} \rightarrow \mathrm{D}$ link is used in the current transmission block, the destination compares the current $\mathrm{SNR} \gamma_{\mathrm{R}_{1}}$ with a preselected threshold $\kappa$. The branch switching occurs when

$$
\gamma_{\mathrm{e} 2 \mathrm{e}, \mathrm{R}_{1}}^{\mathrm{DF}}=\min \left(\gamma_{\mathrm{SR}_{1}}, \gamma_{\mathrm{R}_{1} \mathrm{D}}\right)<\kappa,
$$

for the case of DF, and

$$
\gamma_{\mathrm{e} 2 \mathrm{e}, \mathrm{R}_{1}}^{\mathrm{AF}}=\frac{\gamma_{\mathrm{SR}_{1}} \gamma_{\mathrm{R}_{1} \mathrm{D}}}{\gamma_{\mathrm{SR}_{1}}+\gamma_{\mathrm{R}_{1} \mathrm{D}}+1}<\kappa
$$

for the case of AF.

Note that $\gamma_{\mathrm{e} 2 \mathrm{e}, \mathrm{R}_{1}}^{\mathrm{DF}}$ and $\gamma_{\mathrm{e} 2 \mathrm{e}, \mathrm{R}_{1}}^{\mathrm{AF}}$ are the end-to-end SNRs of relay $R_{1}$ when relay $R_{1}$ uses $D F$ and AF protocols, respectively.

Otherwise, the $S \rightarrow R_{1} \rightarrow$ D link continues as the active link for information transmission in the next block time $T$. Similarly, when the $S \rightarrow R_{2} \rightarrow$ D link is activated in the current transmission block, the branch switching occurs when

$$
\gamma_{\mathrm{e} 2 \mathrm{e}, \mathrm{R}_{2}}^{\mathrm{DF}}=\min \left(\gamma_{\mathrm{SR}_{2}}, \gamma_{\mathrm{R}_{2} \mathrm{D}}\right)<\kappa,
$$

for the case of DF and

$$
\gamma_{\mathrm{e} 2 \mathrm{e}, \mathrm{R}_{2}}^{\mathrm{AF}}=\frac{\gamma_{\mathrm{SR}_{2}} \gamma_{\mathrm{R}_{2} \mathrm{D}}}{\gamma_{\mathrm{SR}_{2}}+\gamma_{\mathrm{R}_{2} \mathrm{D}}+1}<\kappa
$$

for the case of AF.

Note that $\gamma_{\mathrm{e} 2 \mathrm{e}, \mathrm{R}_{2}}^{\mathrm{DF}}$ and $\gamma_{\mathrm{e} 2 \mathrm{e}, \mathrm{R}_{2}}^{\mathrm{AF}}$ are the end-to-end SNRs of relay $R_{2}$ when relay $R_{2}$ uses $D F$ and AF protocols, respectively.

The switching process will be implemented by appropriate feedback sent from the destination to the source and relays, indicating a switching on the transmission path.

\section{Performance analysis}

In this section, we will study the performance analysis of the considered system in terms of outage probability. Depending on AF or DF protocol which is used at relays, we need to study outage probability of the proposed system in a separated manner.

\subsection{Two relays operate in DF mode}

We first derive the system outage probability when two relays operate in DF mode. With DF protocol using at two relays, the signal reaching the destination has to be demodulated two times, i.e., at the relay and at the 
destination. To characterize the system outage probability, we need to evaluate the steady state selection probability of each activating link, which is defined as the proportion of time that link will be used as the active link. Let us denote the steady state selection probability of $\mathrm{S} \rightarrow$ $\mathrm{R}_{1} \rightarrow \mathrm{D}$ link and $\mathrm{S} \rightarrow \mathrm{R}_{2} \rightarrow \mathrm{D}$ link are $p_{\mathrm{R}_{1}}$ and $p_{\mathrm{R}_{2}}$, respectively, which have been derived in ([28], Eqs. (6) and (7)) as

$$
\begin{gathered}
p_{\mathrm{R}_{1}}=\frac{F_{\gamma_{\mathrm{e} 2, \mathrm{R}_{2}}^{\mathrm{DF}}}(\kappa)}{F_{\gamma_{\mathrm{e} 2 e, \mathrm{R}_{1}}^{\mathrm{DF}}}(\kappa)+F_{\gamma_{\mathrm{e} 2 e, \mathrm{R}_{2}}^{\mathrm{DF}}}(\kappa)}, \\
p_{\mathrm{R}_{2}}=\frac{F_{\gamma_{\mathrm{e} 2 e, \mathrm{R}_{1}}^{\mathrm{DF}}}(\kappa)}{F_{\gamma_{\mathrm{e} 2 e, \mathrm{R}_{1}}^{\mathrm{DP}}}(\kappa)+F_{\gamma_{\mathrm{e} 2 e, \mathrm{R}_{2}}^{\mathrm{DF}}}(\kappa)},
\end{gathered}
$$

where $F_{\gamma_{\mathrm{e} 2 e, \mathrm{R}_{1}}^{\mathrm{DF}}}(\kappa)$ and $F_{\gamma_{\mathrm{e} 2, \mathrm{R}}^{\mathrm{DF}}}(\kappa)$ are the cumulative distribution functions (CDFs) of $\gamma_{\mathrm{e} 2 \mathrm{e}, \mathrm{R}_{1}}^{\mathrm{DF}}$ and $\gamma_{\mathrm{e} 2 \mathrm{e}, \mathrm{R}_{2}}^{\mathrm{DF}}$ evaluated at the preselected switching threshold $\kappa$, respectively.

To calculate (23) and (24), we first need to derive the cumulative distribution functions (CDFs) of $\gamma_{\mathrm{e} 2 \mathrm{e}, \mathrm{R}_{1}}^{\mathrm{DF}}$ and $\gamma_{\mathrm{e} 2 \mathrm{e}, \mathrm{R}_{2}}^{\mathrm{DF}}$ evaluated at the preselected switching threshold $\kappa$.

Lemma $1 F_{\gamma_{\mathrm{e} 2, \mathrm{R}_{1}}^{\mathrm{DF}}}(\kappa)$ is given by

$$
\begin{aligned}
F_{\gamma_{\mathrm{e} 2, \mathrm{R}_{1}}^{\mathrm{DF}}}(\kappa) & \approx 1-e^{-\frac{\kappa d_{\mathrm{SR}_{1}}^{m}}{\bar{\gamma} h_{\mathrm{SR}_{1}}}} \\
+ & \frac{\kappa d_{\mathrm{R}_{1} \mathrm{D}}^{m} \Gamma\left(0, \frac{\kappa d_{\mathrm{SR}_{1}}}{\bar{\gamma} h_{\mathrm{SR}_{1}}}\right)\left(d_{\mathrm{SR}_{1}}^{m}-\eta \lambda_{\mathrm{f}_{1}}\right)}{\eta \bar{\gamma} \lambda_{h_{1} \mathrm{D}}{ }^{\lambda} h_{\mathrm{SR}_{1}}} .
\end{aligned}
$$

Proof See Appendix.

Similarly, we also obtain $F_{\gamma_{\mathrm{e} 2 \mathrm{R}, \mathrm{R}_{2}}^{\mathrm{DF}}}(\kappa)$, which can be expressed as

$$
\begin{aligned}
F_{\gamma_{\mathrm{e} 2}, \mathrm{R}_{2}}^{\mathrm{DF}} & (\kappa) \approx 1-e^{-\frac{\kappa d_{\mathrm{SR}_{2}}^{m}}{\bar{\gamma} h_{\mathrm{SR}_{2}}}} \\
+ & \frac{\kappa d_{\mathrm{R}_{2} \mathrm{D}}^{m} \Gamma\left(0, \frac{\kappa d_{\mathrm{SR}_{2}}}{\bar{\gamma} \lambda h_{\mathrm{R}_{2}}}\right)\left(d_{\mathrm{SR}_{2}}^{m}-\eta \lambda_{\mathrm{fR}_{2}}\right)}{\eta \bar{\gamma} \lambda_{h_{R_{2}} \mathrm{D}} \lambda_{h \mathrm{SR}_{2}}} .
\end{aligned}
$$

By plugging (25) and (26) into (23) and (24), we will obtain the steady state selection probabilities of $\mathrm{S} \rightarrow$ $\mathrm{R}_{1} \rightarrow \mathrm{D}$ link and $\mathrm{S} \rightarrow \mathrm{R}_{2} \rightarrow \mathrm{D}$ link.

Next, we study outage probability of our proposed system. Using the law of total probability and the steady state selection probability of two links, which is derived in Eqs. (23) and (24), the outage probability of the system is given by

$$
\begin{aligned}
& \mathrm{P}_{\text {out }}^{\mathrm{DF}}\left(\gamma_{\text {th }}\right)=p_{\mathrm{R}_{1}}\left[\left(1-F_{\gamma_{\mathrm{e} 2, \mathrm{R}_{1}}^{\mathrm{DF}}}(\kappa)\right) \operatorname{Pr}\left(\gamma_{\mathrm{e} 2 \mathrm{e}, \mathrm{R}_{1}}^{\mathrm{DF}} \leq \gamma_{\mathrm{th}} \mid \gamma_{\mathrm{e} 2 \mathrm{e}, \mathrm{R}_{1}}^{\mathrm{DF}}>\kappa\right)\right. \\
& \left.+F_{\gamma_{\mathrm{e} 2 \mathrm{e}, \mathrm{R}_{1}}^{\mathrm{DF}}}(\kappa) \operatorname{Pr}\left(\gamma_{\mathrm{e} 2 \mathrm{e}, \mathrm{R}_{2}}^{\mathrm{DF}} \leq \gamma_{\mathrm{th}} \mid \gamma_{\mathrm{e} 2 \mathrm{e}, \mathrm{R}_{1}}^{\mathrm{DF}} \leq \kappa\right)\right] \\
& +p_{\mathrm{R}_{2}}\left[\left(1-F_{\gamma_{\mathrm{ee}, \mathrm{R}_{2}}^{\mathrm{DF}}}(\kappa)\right) \operatorname{Pr}\left\{\gamma_{\mathrm{e} 2 \mathrm{e}, \mathrm{R}_{2}}^{\mathrm{DF}} \leq \gamma_{\mathrm{th}} \mid \gamma_{\mathrm{e} 2 e, \mathrm{R}_{2}}^{\mathrm{DF}}>\kappa\right\}\right. \\
& \left.+F_{\gamma_{\mathrm{e} 2, \mathbb{R}_{2}}^{\mathrm{DF}}}(\kappa) \operatorname{Pr}\left(\gamma_{\mathrm{e} 2 \mathrm{e}, \mathrm{R}_{1}}^{\mathrm{DF}} \leq \gamma_{\mathrm{th}} \mid \gamma_{\mathrm{e} 2 e, \mathrm{R}_{2}}^{\mathrm{DF}} \leq \kappa\right)\right] \text {. }
\end{aligned}
$$

Then, we need to derive the conditional outage probabilities, which can be expressed under the general form as follows

$$
\operatorname{Pr}\left\{\gamma_{M} \leq \gamma_{\mathrm{th}} \mid \gamma_{M}>t\right\}= \begin{cases}\frac{F_{\gamma_{M}}\left(\gamma_{\mathrm{th}}\right)-F_{\gamma_{M}}(t)}{1-F_{\gamma_{M}}(t)}, & t \leq \gamma_{\mathrm{th}} \\ 0, & t>\gamma_{\mathrm{th}}\end{cases}
$$

where conditional outage probabilities in (27) can be obtained by replacing $\gamma_{M}$ and $t$ in (28) with the suitable values, i.e., $\gamma_{M} \in\left\{\gamma_{\mathrm{e} 2 \mathrm{e}, \mathrm{R}_{1}}^{\mathrm{DF}}, \gamma_{\mathrm{e} 2 \mathrm{e}, \mathrm{R}_{2}}^{\mathrm{DF}}\right\}$ and $t \in\{\kappa, 0\}$. In this paper, we adopt the cumulative distribution function (CDF) of $\gamma_{M}$ evaluated at the outage threshold SNR $\gamma_{\mathrm{th}}$, which is expressed as $\gamma_{\mathrm{th}}=2^{r}-1$ with $r$ representing the target rate, coincides with the conditional outage probability when $M$ link is selected with $M \in\left\{\mathrm{R}_{1}, \mathrm{R}_{2}\right\}$, it means that $F_{\gamma_{M}}\left(\gamma_{\text {th }}\right)=\operatorname{Pr}\left\{\gamma_{M} \leq \gamma_{\text {th }} \mid\left(\gamma_{M}>0\right)\right\}$ and $F_{\gamma_{M}}(t)=$ $\operatorname{Pr}\left(\gamma_{M}<t\right)=1-\operatorname{Pr}\left(\gamma_{M}>t\right)$. We note that since $\gamma_{\mathrm{e} 2 \mathrm{e}, \mathrm{R}_{1}}^{\mathrm{DF}}$ and $\gamma_{\mathrm{e} 2 \mathrm{e}, \mathrm{R}_{2}}^{\mathrm{DF}}$ are independent, so $\operatorname{Pr}\left(\gamma_{\mathrm{e} 2 \mathrm{e}, \mathrm{R}_{2}}^{\mathrm{DF}} \leq \gamma_{\mathrm{th}} \mid \gamma_{\mathrm{e} 2 \mathrm{e}, \mathrm{R}_{1}}^{\mathrm{DF}} \leq\right.$ $\kappa)=F_{\gamma_{\mathrm{e} 2 \mathrm{e}, \mathrm{R}_{2}}}\left(\gamma_{\mathrm{th}}\right)$ and $\operatorname{Pr}\left(\gamma_{\mathrm{e} 2 \mathrm{e}, \mathrm{R}_{1}}^{\mathrm{DF}} \leq \gamma_{\mathrm{th}} \mid \gamma_{\mathrm{e} 2 \mathrm{e}, \mathrm{R}_{2}}^{\mathrm{DF}} \leq \kappa\right)=$ $F_{\gamma_{\mathrm{e} 2 \mathrm{e}, \mathrm{R}_{1}}^{\mathrm{DF}}}\left(\gamma_{\mathrm{th}}\right)$.

By plugging conditional outage probabilities (which are calculated by (28)) and state selection probabilities (which are derived in (23) and (24)) into (27), we obtain the system outage probability as in (30) (on top of the next page) with $F_{\gamma_{\mathrm{e} 2 e, \mathrm{R}_{1}}^{\mathrm{DF}}}\left(\gamma_{\mathrm{th}}\right)$ and $F_{\gamma_{\mathrm{e} 2 \mathrm{e}, \mathrm{R}_{2}}^{\mathrm{DF}}}\left(\gamma_{\mathrm{th}}\right)$ can be calculated by replacing $\kappa$ in (25) and (26) with $\gamma_{\text {th }}$.

From (30), it is obvious that choosing preselected threshold $\kappa$ will affect the outage probability of system. Noting that the system outage probability in (30) is minimized when $\gamma_{t h}=\kappa$, this will be validated by simulation result in Section 6. Therefore, $\mathrm{P}_{\text {out }}^{\mathrm{DF}}\left(\gamma_{\text {th }}\right)$ in this case can be expressed as

$$
\mathrm{P}_{\text {out }}^{\mathrm{DF}}\left(\gamma_{\mathrm{th}}\right)=F_{\gamma_{\mathrm{e} 2, \mathrm{R}_{1}}^{\mathrm{DF}}}\left(\gamma_{\mathrm{th}}\right) F_{\gamma_{\mathrm{e} 2 \mathrm{e}, \mathrm{R}_{2}}^{\mathrm{DF}}}\left(\gamma_{\mathrm{th}}\right) .
$$

We can see that the minimum outage probability in (29) is equal to the outage probability of the system which selects the better link between $\mathrm{S} \rightarrow \mathrm{R}_{1} \rightarrow \mathrm{D}$ and $\mathrm{S} \rightarrow$ $\mathrm{R}_{2} \rightarrow \mathrm{D}$ link for source information transmission.

\subsection{Two relays operate in AF mode}

Let us now consider that both relays use AF protocol to forward the source information to the destination. The corresponding SNRs of two branches are given on the top of next page, 


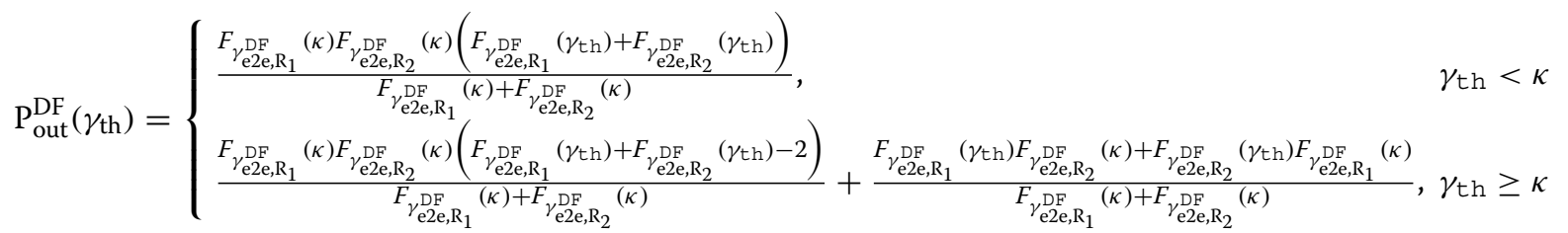

$$
\begin{aligned}
& \gamma_{\mathrm{e} 2 \mathrm{e}, \mathrm{R}_{1}}^{\mathrm{AF}}=\frac{\gamma_{\mathrm{SR}_{1}} \gamma_{\mathrm{R}_{1} \mathrm{D}}}{\gamma_{\mathrm{SR}_{1}}+\gamma_{\mathrm{R}_{1} \mathrm{D}}+1}=\frac{\eta \bar{\gamma}^{2}\left|h_{\mathrm{SR}_{1}}\right|^{4}\left|h_{\mathrm{R}_{1} \mathrm{D}}\right|^{2}}{\left|h_{\mathrm{SR}_{1}}\right|^{2} \bar{\gamma} d_{\mathrm{R}_{1} \mathrm{D}}^{m}\left(d_{\mathrm{SR}_{1}}^{m}-\eta\left|f_{\mathrm{R}_{1}}\right|^{2}\right)+\left|h_{\mathrm{R}_{1} \mathrm{D}}\right|^{2} \eta\left|h_{\mathrm{SR}_{1}}\right|^{2} \bar{\gamma} d_{\mathrm{SR}_{1}}^{m}+d_{\mathrm{SR}_{1}}^{m} d_{\mathrm{R}_{1} \mathrm{D}}^{m}\left(d_{\mathrm{SR}_{1}}^{m}-\eta\left|f_{\mathrm{R}_{1}}\right|^{2}\right)} . \\
& \gamma_{\mathrm{e} 2 \mathrm{e}, \mathrm{R}_{2}}^{\mathrm{AF}}=\frac{\gamma_{\mathrm{SR}_{2}} \gamma_{\mathrm{R}_{2} \mathrm{D}}}{\gamma_{\mathrm{SR}_{2}}+\gamma_{\mathrm{R}_{2} \mathrm{D}}+1}=\frac{\eta \bar{\gamma}^{2}\left|h_{\mathrm{SR}_{2}}\right|^{4}\left|h_{\mathrm{R}_{2} \mathrm{D}}\right|^{2}}{\left|h_{\mathrm{SR}_{2}}\right|^{2} \bar{\gamma} d_{\mathrm{R}_{2} \mathrm{D}}^{m}\left(d_{\mathrm{SR}_{2}}^{m}-\eta\left|f_{\mathrm{R}_{2}}\right|^{2}\right)+\left|h_{\mathrm{R}_{2} \mathrm{D}}\right|^{2} \eta\left|h_{\mathrm{SR}_{2}}\right|^{2} \bar{\gamma} d_{\mathrm{SR}_{2}}^{m}+d_{\mathrm{SR}_{2}}^{m} d_{\mathrm{R}_{2} \mathrm{D}}^{m}\left(d_{\mathrm{SR}_{2}}^{m}-\eta\left|f_{\mathrm{R}_{2}}\right|^{2}\right)} . \\
& F_{\gamma_{\mathrm{e} 2, \mathrm{R}_{1}}^{\mathrm{AP}}}(\kappa)=\operatorname{Pr}\left(\frac{\eta \bar{\gamma}^{2}\left|h_{\mathrm{SR}_{1}}\right|^{4}\left|h_{\mathrm{R}_{1} \mathrm{D}}\right|^{2}}{\left|h_{\mathrm{SR}_{1}}\right|^{2} \bar{\gamma} d_{\mathrm{R}_{1} \mathrm{D}}^{m}\left(d_{\mathrm{SR}_{1}}^{m}-\eta\left|f_{\mathrm{R}_{1}}\right|^{2}\right)+\left|h_{\mathrm{R}_{1} \mathrm{D}}\right|^{2} \eta\left|h_{\mathrm{SR}_{1}}\right|^{2} \bar{\gamma} d_{\mathrm{SR}_{1}}^{m}+d_{\mathrm{SR}_{1}}^{m} d_{\mathrm{R}_{1} \mathrm{D}}^{m}\left(d_{\mathrm{SR}_{1}}^{m}-\eta\left|f_{\mathrm{R}_{1}}\right|^{2}\right)}<\kappa\right) .
\end{aligned}
$$

where the last results in (31) and (32) are obtained by plugging (16) and (18) into (9) and (14) and using (2) and (4).

Next, we need to derive the cumulative distribution functions (CDFs) of $\gamma_{\mathrm{e} 2 \mathrm{e}, \mathrm{R}_{1}}^{\mathrm{AF}}$ and $\gamma_{\mathrm{e} 2 \mathrm{e}, \mathrm{R}_{2}}^{\mathrm{AF}}$ evaluated at the predesigned switching threshold $\kappa \cdot F_{\gamma_{\mathrm{e} 2 \mathrm{AF}, \mathrm{R}_{1}}^{\mathrm{AF}}}(\kappa)$ is expressed as follows in (33).

Lemma $2 F_{\gamma_{\mathrm{e} 2 \mathrm{~A}, \mathrm{R}_{1}}^{\mathrm{AP}}}(\kappa)$ is given by

$$
\begin{aligned}
F_{\gamma_{\mathrm{e} 2 \mathrm{R}, \mathrm{R}_{1}}^{\mathrm{AF}}}(\kappa) \approx & 1-e^{-\frac{\kappa d_{\mathrm{SR}_{1}}^{m}}{\bar{\gamma} h_{\mathrm{SR}_{1}}} \int_{0}^{\infty} \sqrt{\frac{4 \kappa d_{\mathrm{R}_{1} \mathrm{D}}^{m}\left(d_{\mathrm{SR}_{1}}^{m}-\eta u\right)}{\lambda_{h_{\mathrm{S}_{1}}} \eta \bar{\gamma} \lambda_{\mathrm{R}_{1} \mathrm{D}}}}} \\
& \times K_{1}\left(\sqrt{\frac{4 \kappa d_{\mathrm{R}_{1} \mathrm{D}}^{m}\left(d_{\mathrm{SR}_{1}}^{m}-\eta u\right)}{\lambda_{h_{\mathrm{SR}_{1}}} \eta \bar{\gamma} \lambda_{h_{\mathrm{R}_{1} \mathrm{D}}}}}\right) \frac{1}{\lambda_{\mathrm{R}_{1}}} e^{-\frac{u}{\lambda_{\mathrm{R}_{1}}}} d u .
\end{aligned}
$$

Proof See Appendix.

Similarly, we also obtain $F_{\gamma_{\mathrm{e} 2 \mathrm{~A}, \mathrm{R}_{2}}^{\mathrm{AF}}}(\kappa)$, which can be expressed as

$$
\begin{aligned}
F_{\gamma_{\mathrm{e} 2, \mathrm{R}_{2}}^{\mathrm{AF}}}(\kappa) \approx & 1-e^{-\frac{\kappa d_{\mathrm{SR}_{2}}^{m}}{\bar{\gamma} h_{\mathrm{SR}_{2}}} \int_{0}^{\infty} \sqrt{\frac{4 \kappa d_{\mathrm{R}_{2} \mathrm{D}}^{m}\left(d_{\mathrm{SR}_{2}}^{m}-\eta u\right)}{\lambda_{h_{\mathrm{S}_{2}}} \eta \bar{\gamma} \lambda_{\mathrm{R}_{2} \mathrm{D}}}}} \\
& \times K_{1}\left(\sqrt{\frac{4 \kappa d_{\mathrm{R}_{2} \mathrm{D}}^{m}\left(d_{\mathrm{SR}_{2}}^{m}-\eta u\right)}{\lambda_{h_{\mathrm{SR}_{2}}} \eta \bar{\gamma} \lambda_{h_{\mathrm{R}_{2}} \mathrm{D}}}}\right) \frac{1}{\lambda_{\mathrm{R}_{2}}} e^{-\frac{u}{\lambda_{\mathrm{R}_{2}}}} d u .
\end{aligned}
$$

Similar to the DF case, the system outage probability for AF mode is obtained directly from Eq. (30) by plugging (34) and (35) into (30), which can be expressed as follows.

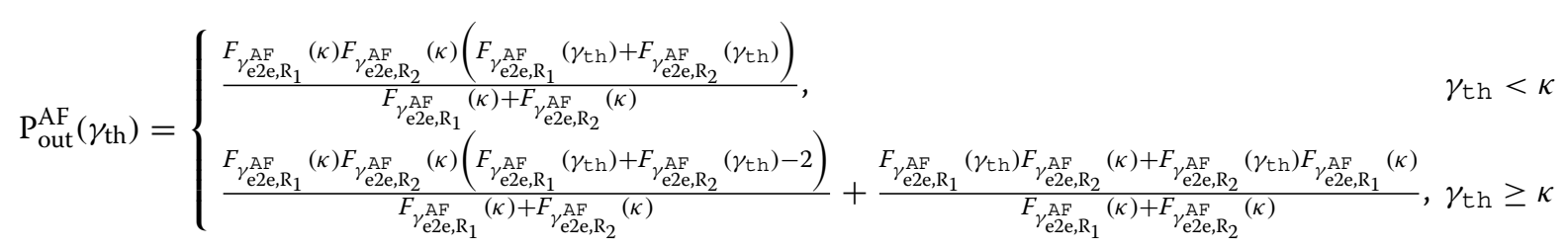


Noting that $F_{\gamma_{\mathrm{e} 2 \mathrm{e}, \mathrm{R}_{1}}^{\mathrm{AF}}}\left(\gamma_{\mathrm{th}}\right)$ and $F_{\gamma_{\mathrm{e} 2 \mathrm{e}, \mathrm{R}_{2}}^{\mathrm{AF}}}\left(\gamma_{\mathrm{th}}\right)$ can also be calculated by replacing $\kappa$ in (34) and (35) with $\gamma_{\mathrm{th}}$. In the case of $\gamma_{\mathrm{th}}=\kappa$, the system outage probability is also minimized and calculated as in (29), which can be derived as follows (this will be validated by simulation result in Section 6)

$$
\mathrm{P}_{\text {out }}^{\mathrm{AF}}\left(\gamma_{\mathrm{th}}\right)=F_{\gamma_{\mathrm{e} 2 \mathrm{e}, \mathrm{R}_{1}}^{\mathrm{AF}}}\left(\gamma_{\mathrm{th}}\right) F_{\gamma_{\mathrm{e} 2 \mathrm{e}, \mathrm{R}_{2}}^{\mathrm{AF}}}\left(\gamma_{\mathrm{th}}\right)
$$

We can see that the minimum outage probability in (37) is equal to the outage probability of the system which selects the better link between $S \rightarrow R_{1} \rightarrow D$ and $S \rightarrow$ $\mathrm{R}_{2} \rightarrow \mathrm{D}$ link for source information transmission.

\section{Diversity analysis}

In order to present insights into the obtained system outage probabilities, the diversity analysis of DF and AF cases is examined. The diversity gain, D, achieved by a cooperative scheme can be shown as $[29,30]$

$$
D=-\lim _{\gamma \rightarrow \infty} \frac{\log P_{\text {out }}(\bar{\gamma})}{\log \bar{\gamma}}
$$

\subsection{DF case}

Considering at the high $\operatorname{SNR}(\bar{\gamma} \rightarrow \infty)$, using $\left(e^{-x} \approx\right.$ $1-x)$ and $\Gamma(0, x)=-\operatorname{Ei}(-x) \approx-C_{E}-\ln (x)([34]$, Eq. (8.359.1) and Eq. (8.214.1)) when $x \rightarrow 0^{+}$, where $C_{E}$ denotes the Euler's constant ([34], Eq. (8.367.1)), (25) can be derived as

$$
\begin{aligned}
F_{\gamma_{\mathrm{e} 2, \mathrm{R}_{1}}^{\mathrm{DF}}}(\kappa) & \approx 1-\left(1-\frac{\kappa d_{\mathrm{SR}_{1}}^{m}}{\bar{\gamma} \lambda_{h_{\mathrm{SR}_{1}}}}\right) \\
& -\frac{\kappa d_{\mathrm{R}_{1} \mathrm{D}}^{m}\left(C_{E}+\ln \left(\frac{\kappa d_{\mathrm{R}_{1}}^{m}}{\bar{\gamma} h_{\mathrm{SR}_{1}}}\right)\right)\left(d_{\mathrm{SR}_{1}}^{m}-\eta \lambda_{f_{\mathrm{R}_{1}}}\right)}{\eta \bar{\gamma} \lambda_{h_{\mathrm{R}_{1} \mathrm{D}}} \lambda_{h_{\mathrm{SR}_{1}}}} \\
& \approx \frac{\kappa d_{\mathrm{SR}_{1}}^{m}}{\bar{\gamma} \lambda_{h_{\mathrm{SR}_{1}}}}-\frac{\kappa d_{\mathrm{R}_{1} \mathrm{D}}^{m}\left(C_{E}+\ln \left(\frac{\kappa d_{\mathrm{SR}_{1}}^{m}}{\bar{\gamma} h_{\mathrm{SR}_{1}}}\right)\right)\left(d_{\mathrm{SR}_{1}}^{m}-\eta \lambda_{f_{\mathrm{R}_{1}}}\right)}{\eta \bar{\gamma} \lambda_{h_{\mathrm{R}_{1} \mathrm{D}}} \lambda_{h_{\mathrm{SR}_{1}}}} .
\end{aligned}
$$

Similarly, $F_{\gamma_{\mathrm{e} 2 \mathrm{e}, \mathrm{R}_{2}}^{\mathrm{DF}}}(\kappa)$ is given by

$$
F_{\gamma_{\mathrm{e} 2 \mathrm{R}, \mathrm{R}_{2}}^{\mathrm{DF}}}(\kappa) \approx \frac{\kappa d_{\mathrm{SR}_{2}}^{m}}{\bar{\gamma} \lambda_{h_{\mathrm{SR}_{2}}}}-\frac{\kappa d_{\mathrm{R}_{2} \mathrm{D}}^{m}\left(C_{E}+\ln \left(\frac{\kappa d_{\mathrm{SR}_{2}}^{m}}{\bar{\gamma} \lambda_{\mathrm{SR}_{2}}}\right)\right)\left(d_{\mathrm{SR}_{2}}^{m}-\eta \lambda_{\mathrm{R}_{2}}\right)}{\eta \bar{\gamma} \lambda_{h_{\mathrm{R}_{2} \mathrm{D}}} \lambda_{h_{\mathrm{SR}_{2}}}} .
$$

Denoting $\alpha_{1}=\frac{d_{\mathrm{SR}_{1}}^{m}}{\lambda_{h_{\mathrm{SR}_{1}}}}, \xi_{1}=\frac{d_{\mathrm{R}_{1} \mathrm{D}}^{m}}{\eta \lambda_{h_{\mathrm{R}_{1} \mathrm{D}}} \lambda_{h_{\mathrm{SR}_{1}}}}, \chi_{1}=d_{\mathrm{SR}_{1}}^{m}-$ $\eta \lambda_{f_{\mathrm{R}_{1}}}, \alpha_{2}=\frac{d_{\mathrm{SR}_{2}}^{m}}{\lambda_{h_{\mathrm{SR}_{2}}}}, \xi_{2}=\frac{d_{\mathrm{R}_{2} \mathrm{D}}^{m}}{\eta \lambda_{h_{\mathrm{R}_{2} \mathrm{D}}} \lambda_{h_{\mathrm{SR}_{2}}}}$ and $\chi_{2}=d_{\mathrm{SR}_{2}}^{m}-\eta \lambda_{f_{\mathrm{R}_{2}}}$, (39) and (40) can be rewritten as

$$
\begin{aligned}
F_{\gamma_{\mathrm{e} 2 \mathrm{e}, \mathrm{R}_{1}}^{\mathrm{DF}}}(\kappa) & \approx \frac{\kappa \alpha_{1}}{\bar{\gamma}}-\frac{\kappa \xi_{1}\left(C_{E}+\ln \left(\frac{\kappa \alpha_{1}}{\bar{\gamma}}\right)\right) \chi_{1}}{\bar{\gamma}} \\
& \approx \frac{\kappa}{\bar{\gamma}}\left(\alpha_{1}-\xi_{1} C_{E} \chi_{1}-\xi_{1} \ln \left(\frac{\kappa \alpha_{1}}{\bar{\gamma}}\right) \chi_{1}\right) \\
& \approx \frac{\kappa}{\bar{\gamma}}\left(\zeta_{1}-\nu_{1} \ln \left(\frac{\kappa \alpha_{1}}{\bar{\gamma}}\right)\right),
\end{aligned}
$$

where $\zeta_{1}=\alpha_{1}-\xi_{1} C_{E} \chi_{1}$ and $\nu_{1}=\xi_{1} \chi_{1}$

$$
\begin{aligned}
F_{\gamma_{\mathrm{e} 2 \mathrm{e}, \mathrm{R}_{2}}^{\mathrm{DF}}}(\kappa) & \approx \frac{\kappa \alpha_{2}}{\bar{\gamma}}-\frac{\kappa \xi_{2}\left(C_{E}+\ln \left(\frac{\kappa \alpha_{2}}{\bar{\gamma}}\right)\right) \chi_{2}}{\bar{\gamma}} \\
& \approx \frac{\kappa}{\bar{\gamma}}\left(\alpha_{2}-\xi_{2} C_{E} \chi_{2}-\xi_{2} \ln \left(\frac{\kappa \alpha_{2}}{\bar{\gamma}}\right) \chi_{2}\right) \\
& \approx \frac{\kappa}{\bar{\gamma}}\left(\zeta_{2}-\nu_{2} \ln \left(\frac{\kappa \alpha_{2}}{\bar{\gamma}}\right)\right),
\end{aligned}
$$

where $\zeta_{2}=\alpha_{2}-\xi_{2} C_{E} \chi_{2}$ and $\nu_{2}=\xi_{2} \chi_{2}$

For the case of $\gamma_{\text {th }}<\kappa, \mathrm{P}_{\text {out }}^{\mathrm{DF}}\left(\gamma_{\text {th }}\right)$ can be given by

$$
\begin{aligned}
& \mathrm{P}_{\text {out }}^{\mathrm{DF}}\left(\gamma_{\mathrm{th}}\right)=\frac{F_{\gamma_{\mathrm{e} 2 \mathrm{e}, \mathrm{R}_{1}}^{\mathrm{DF}}}(\kappa) F_{\gamma_{\mathrm{e} 2 \mathrm{e}, \mathrm{R}_{2}}^{\mathrm{DF}}}(\kappa)\left(F_{\gamma_{\mathrm{e} 2 \mathrm{e}, \mathrm{R}_{1}}^{\mathrm{DF}}}\left(\gamma_{\mathrm{th}}\right)+F_{\gamma_{\mathrm{e} 2 \mathrm{e}, \mathrm{R}_{2}}^{\mathrm{DF}}}\left(\gamma_{\mathrm{th}}\right)\right)}{F_{\gamma_{\mathrm{e} 2 \mathrm{e}, \mathrm{R}_{1}}^{\mathrm{DF}}}(\kappa)+F_{\gamma_{\mathrm{e} 2 \mathrm{e}, \mathrm{R}_{2}}^{\mathrm{DF}}}(\kappa)} \\
& =\frac{\left(\frac{\kappa \gamma_{\mathrm{th}}}{\bar{\gamma}^{2}}\right)\left(\zeta_{1}-v_{1} \ln \left(\frac{\kappa \alpha_{1}}{\bar{\gamma}}\right)\right)\left(\zeta_{2}-v_{2} \ln \left(\frac{\kappa \alpha_{2}}{\bar{\gamma}}\right)\right)}{\left(\zeta_{1}-v_{1} \ln \left(\frac{\kappa \alpha_{1}}{\bar{\gamma}}\right)+\zeta_{2}-\nu_{2} \ln \left(\frac{\kappa \alpha_{2}}{\bar{\gamma}}\right)\right)} \\
& \times\left(\zeta_{1}-v_{1} \ln \left(\frac{\gamma_{\operatorname{th}} \alpha_{1}}{\bar{\gamma}}\right)+\zeta_{2}-v_{2} \ln \left(\frac{\gamma_{\operatorname{th}} \alpha_{2}}{\bar{\gamma}}\right)\right) \text {. }
\end{aligned}
$$

From (38) and (43), we obtain the limitation straightly as follows

$$
\lim _{\bar{\gamma} \rightarrow \infty} \frac{\log \mathrm{P}_{\text {out }}^{\mathrm{DF}}\left(\gamma_{\mathrm{th}}\right)}{\log \bar{\gamma}}=-2 .
$$

When $\gamma_{\text {th }}>\kappa, \mathrm{P}_{\text {out }}^{\mathrm{DF}}\left(\gamma_{\text {th }}\right)$ can be written as

$$
\begin{aligned}
& \mathrm{P}_{\text {out }}^{\mathrm{DF}}\left(\gamma_{\mathrm{th}}\right)=\frac{F_{\gamma_{\mathrm{e} 2 \mathrm{e}, \mathrm{R}_{1}}^{\mathrm{DF}}}(\kappa) F_{\gamma_{\mathrm{e} 2, \mathrm{R} 2}^{\mathrm{DF}}}(\kappa)\left(F_{\gamma_{\mathrm{e} 2, \mathrm{R}}^{\mathrm{DF}}}\left(\gamma_{\mathrm{th}}\right)+F_{\gamma_{\mathrm{e} 2, \mathrm{R}_{2}}^{\mathrm{DF}}}\left(\gamma_{\mathrm{th}}\right)-2\right)}{F_{\gamma_{\mathrm{e} 2, \mathrm{R}_{1}}^{\mathrm{DF}}}(\kappa)+F_{\gamma_{\mathrm{e} 2, \mathrm{R}_{2}}^{\mathrm{DF}}}(\kappa)} \\
& +\frac{F_{\gamma_{\mathrm{e} 2 e, \mathrm{R}_{1}}^{\mathrm{DF}}}\left(\gamma_{\mathrm{th}}\right) F_{\gamma_{\mathrm{e} 2 e, \mathrm{R}_{2}}^{\mathrm{DF}}}(\kappa)+F_{\gamma_{\mathrm{e} 2, \mathrm{R}_{2}}^{\mathrm{DF}}}\left(\gamma_{\mathrm{th}}\right) F_{\gamma_{\mathrm{e2}, \mathrm{R}_{1}}^{\mathrm{DF}}}(\kappa)}{F_{\gamma_{\mathrm{e} 2, \mathrm{R}_{1}}^{\mathrm{DF}}}(\kappa)+F_{\gamma_{\mathrm{e} 2 e, \mathrm{R}_{2}}^{\mathrm{DF}}}(\kappa)} \\
& =\left[\frac{\left(\frac{\kappa \gamma_{\mathrm{th}}}{\bar{\gamma}^{2}}\right)\left(\zeta_{1}-v_{1} \ln \left(\frac{\kappa \alpha_{1}}{\bar{\gamma}}\right)\right)\left(\zeta_{2}-v_{2} \ln \left(\frac{\kappa \alpha_{2}}{\bar{\gamma}}\right)\right)}{\left(\zeta_{1}-v_{1} \ln \left(\frac{\kappa \alpha_{1}}{\bar{\gamma}}\right)+\zeta_{2}-v_{2} \ln \left(\frac{\kappa \alpha_{2}}{\bar{\gamma}}\right)\right)}\right. \\
& \left.\times\left(\zeta_{1}-v_{1} \ln \left(\frac{\gamma_{\mathrm{th}} \alpha_{1}}{\bar{\gamma}}\right)+\zeta_{2}-v_{2} \ln \left(\frac{\gamma_{\mathrm{th}} \alpha_{2}}{\bar{\gamma}}\right)-\frac{2 \bar{\gamma}}{\gamma_{\mathrm{th}}}\right)\right] \\
& +\frac{1}{\bar{\gamma}}\left[\frac{\left(\gamma_{\operatorname{th}} \zeta_{1}-\gamma_{\operatorname{th}} \nu_{1} \ln \left(\frac{\gamma_{\mathrm{th}} \alpha_{1}}{\bar{\gamma}}\right)\right)\left(\kappa \zeta_{2}-\kappa \nu_{2} \ln \left(\frac{\kappa \alpha_{2}}{\bar{\gamma}}\right)\right)}{\left(\kappa \zeta_{1}-\kappa \nu_{1} \ln \left(\frac{\kappa \alpha_{1}}{\bar{\gamma}}\right)+\kappa \zeta_{2}-\kappa \nu_{2} \ln \left(\frac{\kappa \alpha_{2}}{\bar{\gamma}}\right)\right)}\right. \\
& \left.+\frac{\left(\gamma_{\operatorname{th}} \zeta_{2}-\gamma_{\operatorname{th}} \nu_{2} \ln \left(\frac{\gamma \operatorname{th} \alpha_{2}}{\bar{\gamma}}\right)\right)\left(\kappa \zeta_{1}-\kappa \nu_{1} \ln \left(\frac{\kappa \alpha_{1}}{\bar{\gamma}}\right)\right)}{\left(\kappa \zeta_{1}-\kappa \nu_{1} \ln \left(\frac{\kappa \alpha_{1}}{\bar{\gamma}}\right)+\kappa \zeta_{2}-\kappa \nu_{2} \ln \left(\frac{\kappa \alpha_{2}}{\bar{\gamma}}\right)\right)}\right] .
\end{aligned}
$$

From (38) and (45), it is straightforward to derive the limitation as follows

$$
\lim _{\bar{\gamma} \rightarrow \infty} \frac{\log \mathrm{P}_{\text {out }}^{\mathrm{DF}}\left(\gamma_{\mathrm{th}}\right)}{\log \bar{\gamma}}=-1
$$


In case of $\gamma_{\mathrm{th}}=\kappa, \mathrm{P}_{\text {out }}^{\mathrm{DF}}\left(\gamma_{\mathrm{th}}\right)$ can be shown as

$$
\begin{aligned}
\mathrm{P}_{\mathrm{out}}^{\mathrm{DF}}\left(\gamma_{\mathrm{th}}\right)= & F_{\gamma_{\mathrm{e} 2 \mathrm{e}, \mathrm{R}_{1}}^{\mathrm{DF}}}\left(\gamma_{\mathrm{th}}\right) F_{\gamma_{\mathrm{e} 2 \mathrm{e}, \mathrm{R}_{2}}^{\mathrm{DF}}}\left(\gamma_{\mathrm{th}}\right) \\
= & \left(\frac{\gamma_{\mathrm{th}}}{\bar{\gamma}}\right)^{2}\left(\zeta_{1}-\nu_{1} \ln \left(\frac{\kappa \alpha_{1}}{\bar{\gamma}}\right)\right) \\
& \times\left(\zeta_{2}-\nu_{2} \ln \left(\frac{\kappa \alpha_{2}}{\bar{\gamma}}\right)\right) .
\end{aligned}
$$

From (38) and (47), we obtain the result as follows

$$
\lim _{\bar{\gamma} \rightarrow \infty} \frac{\log \mathrm{P}_{\text {out }}^{\mathrm{DF}}\left(\gamma_{\mathrm{th}}\right)}{\log \bar{\gamma}}=-2 .
$$

From (44), (46), and (48), we can see that the system full diversity can be achieved with order of two when $\kappa \geq \gamma_{\text {th }}$, whereas the diversity order of the system only attains to one when $\kappa<\gamma_{\text {th }}$.

\subsection{AF case}

Since (34) and (35) involve an integral, which in general do not admit closed-form solution, hence, it is not amenable to further processing and diversity order analysis. However, we will conduct diversity order analysis for AF case by using numerical results, which are shown in Fig. 6.

According to [33], the diversity order can be indicated by the slope at high SNR regions of an outage performance curve. As shown in Fig. 6, we can see that the performance curves of two cases $\gamma_{\text {th }}<\kappa$ (corresponds to $\kappa=11$ ) and $\gamma_{\text {th }}=\kappa$ (corresponds to $\kappa=7$ ) are parallel to the curve that is proportional to $\frac{1}{\mathrm{SNR}^{2}}$, which provide the full diversity order while the performance curve of the case $\gamma_{\text {th }}>\kappa$ (corresponds to $\kappa=3$ ) is parallel to the curve that is proportional to $\frac{1}{\mathrm{SNR}}$, which results in the diversity order of one.

\section{HD relaying}

In this section, we consider the existing Power Splittingbased Relaying (PSR) protocol [4], which serves as a benchmark for performance comparison. The purpose of using PSR protocol is that since PSR has a better performance than time switching-based relaying (TSR) protocol, which is shown in [4]. Some of the results have been derived in [4]; however, we present here to make our work self-contained.

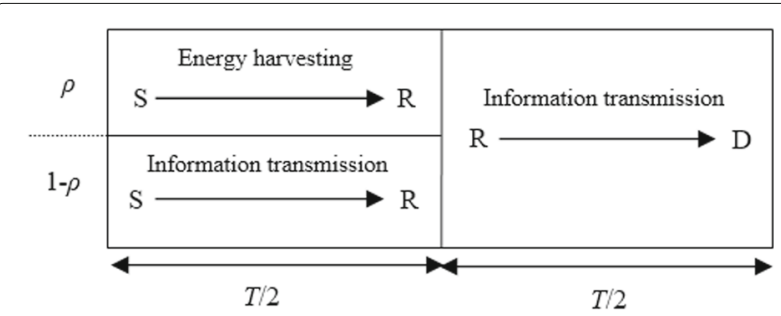

Fig. 3 Power splitting-based wireless-powered relaying [4]
We begin with a brief description of the half-duplex (HD) relaying model. As shown in Fig. 3, the PSR protocol consists of two phases. In the first phase of duration $T / 2$, the source transmits information to the selected relay with the power $\mathcal{P}_{\mathrm{S}}$. The selected relay uses the fraction $\rho$ of the received signal power for energy harvesting and the remaining $(1-\rho)$ portion of the received power for information processing, where $0 \leq \rho \leq 1$. In case of using relay $R_{1}$, the harvested energy at the relay is given by

$$
\mathrm{E}_{\mathrm{R}_{1}}=\frac{\eta \rho \mathcal{P}_{\mathrm{S}}\left|h_{\mathrm{SR}_{1}}\right|^{2}}{d_{\mathrm{SR}_{1}}^{m}} T / 2
$$

Note that, unless otherwise stated, all notations in this section have the same meaning as they have in Subsection 2.2.

Thus the energy scavenged at relay $R_{1}$ is shown as

$$
\mathcal{P}_{\mathrm{R}_{1}}=\frac{\eta \rho \mathcal{P}_{\mathrm{S}}\left|h_{\mathrm{SR}_{1}}\right|^{2}}{d_{\mathrm{SR}_{1}}^{m}} \text {. }
$$

In this HD model, we consider the pessimistic case of PS receiver where the power splitting receiver only utilizes the signal power, but not the antenna noise power, as done in $[31,32]$. The received signal at relay $R_{1}$ for information processing is given by

$$
y_{\mathrm{R}_{1}}=\frac{\sqrt{1-\rho} h_{\mathrm{SR}_{1}}}{\sqrt{d_{\mathrm{SR}_{1}}^{m}}} x_{\mathrm{S}}+n_{\mathrm{R}_{1}} \text {. }
$$

Hence, the instantaneous SNR of $S-R_{1}$ link is shown as

$$
\gamma_{\mathrm{SR}_{1}}=\frac{(1-\rho) \mathcal{P}_{\mathrm{S}}\left|h_{\mathrm{SR}_{1}}\right|^{2}}{d_{\mathrm{SR}_{1}}^{m} N_{0}}=\frac{(1-\rho)\left|h_{\mathrm{SR}_{1}}\right|^{2} \bar{\gamma}}{d_{\mathrm{SR}_{1}}^{m}} \text {. }
$$

In the second phase of duration $T / 2$, using the energy harvested in the first phase, the selected relay forwards the source information to the destination. In case of using

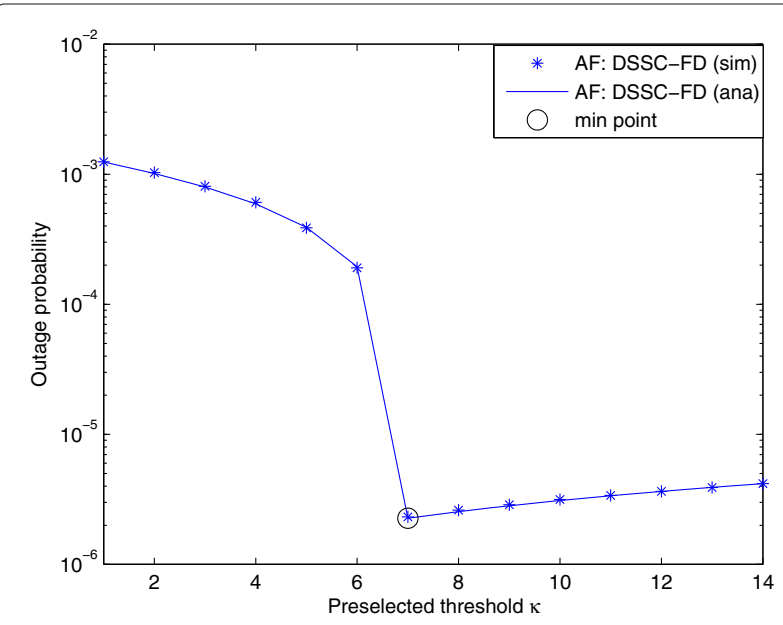

Fig. 4 Outage probability of DSSC full-duplex system with AF protocol versus $\kappa$ when $\gamma_{\text {th }}=7, \bar{\gamma}=50 \mathrm{~dB}$ 
relay $R_{1}$, the received signal at the destination is expressed as

$$
y_{\mathrm{D}}=\frac{h_{\mathrm{R}_{1} \mathrm{D}}}{\sqrt{d_{\mathrm{R}_{1} \mathrm{D}}^{m}}} x_{\mathrm{R}_{1}}+n_{\mathrm{D}}
$$

Hence, the instantaneous SNR of $R_{1}-D$ link is given by

$$
\gamma_{\mathrm{R}_{1} \mathrm{D}}=\frac{\left|h_{\mathrm{R}_{1} \mathrm{D}}\right|^{2} \mathcal{P}_{\mathrm{R}_{1}}}{d_{\mathrm{R}_{1} \mathrm{D}}^{m} N_{0}}=\frac{\left|h_{\mathrm{R}_{1} \mathrm{D}}\right|^{2} \eta \rho \bar{\gamma}\left|h_{\mathrm{SR}_{1}}\right|^{2}}{d_{\mathrm{SR}_{1}}^{m} d_{\mathrm{R}_{1} \mathrm{D}}^{m}},
$$

where the second equality is obtained by using (50) and $\bar{\gamma}=\frac{\mathcal{P}_{\mathrm{S}}}{N_{0}}$.

The system model for the case of using $R_{2}$ is similar to the case of using $R_{1}$, so we skip this description for brevity.

\subsection{DF case}

The end-to-end SNR for HD using DF is presented as (19). Substituting (52) and (54) into (19), $\gamma_{\mathrm{e} 2 \mathrm{e}, \mathrm{R}_{1}}^{\mathrm{DF}}$ is expressed as

$$
\gamma_{\mathrm{e} 2 \mathrm{e}, \mathrm{R}_{1}}^{\mathrm{DF}}=\min \left(\frac{(1-\rho)\left|h_{\mathrm{SR}_{1}}\right|^{2} \bar{\gamma}}{d_{\mathrm{SR}_{1}}^{m}}, \frac{\left|h_{\mathrm{R}_{1} \mathrm{D}}\right|^{2} \eta \rho \bar{\gamma}\left|h_{\mathrm{SR}_{1}}\right|^{2}}{d_{\mathrm{SR}_{1}}^{m} d_{\mathrm{R}_{1} \mathrm{D}}^{m}}\right) .
$$

Lemma $3 F_{\gamma_{\mathrm{e} 2 \mathrm{R}_{1}}^{\mathrm{DF}}}(\kappa)$ is given by

Exact analysis

$$
F_{\gamma_{\mathrm{e} 2 \mathrm{D}, \mathrm{R}_{1}}^{\mathrm{DF}}}(\kappa)=1-\frac{1}{\lambda_{h_{\mathrm{SR}_{1}}}} \int_{\nu_{1}}^{\infty} e^{-\frac{x}{h_{h_{\mathrm{R}}}}-\frac{\kappa d_{\mathrm{SR}_{1}}^{m}}{\eta \rho \gamma \lambda_{1}} h_{\mathrm{R}_{1} \mathrm{D}}^{m}} d x,
$$

where $\nu_{1}=\frac{\kappa d_{\mathrm{SR}_{1}}^{m}}{(1-\rho) \bar{\gamma}}$.

Approximation analysis

$$
\begin{aligned}
F_{\gamma_{\mathrm{e} 2, \mathrm{R}_{1}}^{\mathrm{DF}}}(\kappa) \approx & 1-e^{-\frac{\kappa d_{\mathrm{SR}_{1}}^{m}}{(1-\rho) \bar{\gamma} h_{\mathrm{SR}_{1}}}} \\
& +\frac{\kappa d_{\mathrm{SR}_{1}}^{m} d_{\mathrm{R}_{1} \mathrm{D}}^{m} \Gamma\left(0, \frac{\kappa d_{\mathrm{SR}_{1}}^{m}}{(1-\rho) \bar{\gamma} h_{\mathrm{SR}_{1}}}\right)}{\eta \rho \bar{\gamma} \lambda_{h_{\mathrm{R}_{1}} \mathrm{D}}{ }^{\lambda} h_{\mathrm{SR}_{1}}} .
\end{aligned}
$$

Proof The proof follows the same step used in Lemma 1. Thus we skip the proof for HD using DF for brevity.

Similarly, $F_{\gamma_{\mathrm{e} 2 e, \mathrm{R}_{2}}^{\mathrm{DF}}}(\kappa)$ is expressed as

Exact analysis

$$
F_{\gamma_{\mathrm{e} 2 \mathrm{R}, \mathrm{R}_{2}}^{\mathrm{DF}}}(\kappa)=1-\frac{1}{\lambda_{h_{\mathrm{SR}_{2}}}} \int_{\nu_{2}}^{\infty} e^{-\frac{x}{\lambda h_{\mathrm{SR}_{2}}}-\frac{\kappa d_{\mathrm{S}_{2}}^{m}}{\eta \rho \alpha_{2} \lambda R_{R_{2} \mathrm{D}} \mathrm{D}}} d x
$$

where $\nu_{2}=\frac{\kappa d_{\mathrm{SR}_{2}}^{m}}{(1-\rho) \bar{\gamma}}$.
Approximation analysis

$$
\begin{aligned}
F_{\gamma_{\mathrm{e} 2, \mathrm{R}_{2}}^{\mathrm{DF}}}(\kappa) & \approx 1-e^{-\frac{\kappa d_{\mathrm{SR}_{2}}^{m}}{(1-\rho) \bar{\gamma} \lambda h_{\mathrm{SR}_{2}}}} \\
+ & \frac{\kappa d_{\mathrm{SR}_{2}}^{m} d_{\mathrm{R}_{2} \mathrm{D}}^{m} \Gamma\left(0, \frac{\kappa d_{\mathrm{SR}_{2}}}{(1-\rho) \bar{\gamma} h_{\mathrm{SR}_{2}}}\right)}{\eta \rho \bar{\gamma} h_{\mathrm{R}_{2} \mathrm{D}} h_{\mathrm{SR}_{2}}} .
\end{aligned}
$$

The system outage probability $\mathrm{P}_{\text {out }}^{\mathrm{DF}}\left(\gamma_{\text {th }}\right)$ for HD using DF is calculated using (30), where $F_{\gamma_{\mathrm{e} 2 \mathrm{e}, \mathrm{R}_{1}}^{\mathrm{DF}}}(\kappa)$ and $F_{\gamma_{\mathrm{e} 2, \mathrm{R}_{2}}^{\mathrm{DF}}}(\kappa)$ are derived in (57) and (59), $F_{\gamma_{\mathrm{e} 2 e, \mathrm{R}_{1}}^{\mathrm{DF}}}\left(\gamma_{\mathrm{th}}\right)$ and $F_{\gamma_{\mathrm{e} 2 \mathrm{e}, \mathrm{R}_{2}}}\left(\gamma_{\mathrm{th}}\right)$ can be calculated by replacing $\kappa$ in (57) and (59) with $\gamma_{\text {th }}$.

\subsection{AF case}

The end-to-end SNR for HD utilizing AF is expressed in (20). Substituting (52) and (54) into (20), we obtain $\gamma_{\mathrm{e} 2 \mathrm{e}, \mathrm{R}_{1}}^{\mathrm{AF}}$ which is shown as follows.

$$
\frac{\gamma_{\mathrm{e} 2 e, \mathrm{R}_{1}}^{\mathrm{AF}}=}{(1-\rho) \bar{\gamma} d_{\mathrm{SR}_{1}}^{m} d_{\mathrm{R}_{1} \mathrm{D}}^{m}\left|h_{\mathrm{SR}_{1}}\right|^{2}+\eta \rho \bar{\gamma} d_{\mathrm{SR}_{1}}^{m}\left|h_{\mathrm{SR}_{1}}\right|^{2}\left|h_{\mathrm{R}_{1} \mathrm{D}}\right|^{2}+d_{\mathrm{SR}_{1}}^{2 m} d_{\mathrm{R}_{1} \mathrm{D}}^{m}}
$$

This case has been studied in [4] and we present the result here for the purpose of completeness.

$$
\text { Lemma } 4 F_{\gamma_{\mathrm{e} 2, \mathrm{R}_{1}}^{\mathrm{AF}}}(\kappa) \text { can be shown as }
$$

$F_{\gamma_{\mathrm{e} 2, \mathrm{R}_{1}}^{\mathrm{AF}}}(\kappa)=1-\frac{1}{\lambda h_{\mathrm{SR}_{1}}} \int_{z=\nu_{3} / \chi_{3}}^{\infty} e^{-\left(\frac{z}{\lambda_{h_{\mathrm{SR}}}}+\frac{\alpha_{3} z+\xi_{3}}{\left(\chi_{3} z^{2}-\nu_{3} z\right) \lambda_{\mathrm{R}_{1} \mathrm{D}}}\right)} d z$,

where $\alpha_{3}=\kappa(1-\rho) \bar{\gamma} d_{\mathrm{SR}_{1}}^{m} d_{\mathrm{R}_{1} \mathrm{D}}^{m}, \xi_{3}=\kappa d_{\mathrm{SR}_{1}}^{2 m} d_{\mathrm{R}_{1} \mathrm{D}}^{m}, \chi_{3}=$ $(1-\rho) \rho \eta \bar{\gamma}^{2}$ and $\nu_{3}=\eta \bar{\gamma} \rho d_{\mathrm{SR}_{1}}^{m} \kappa$.

Similarly, $F_{\gamma_{\mathrm{e} 2, \mathrm{R}, \mathrm{R}}^{\mathrm{AF}}}(\kappa)$ can be given by

$F_{\gamma_{\mathrm{e} 2, \mathrm{R}_{2}}^{\mathrm{AF}}}(\kappa)=1-\frac{1}{\lambda_{h_{\mathrm{SR}_{2}}}} \int_{z=\nu_{4} / \chi_{4}}^{\infty} e^{-\left(\frac{z}{\lambda_{\mathrm{SR}_{2}}}+\frac{\alpha_{4} z+\xi_{4}}{\left(x_{4} z^{2}-\nu_{4} z\right) \lambda_{\mathrm{R}_{2} \mathrm{D}}}\right)} d z$,

where $\alpha_{4}=\kappa(1-\rho) \bar{\gamma} d_{\mathrm{SR}_{2}}^{m} d_{\mathrm{R}_{2} \mathrm{D}}^{m}, \xi_{4}=\kappa d_{\mathrm{SR}_{2}}^{2 m} d_{\mathrm{R}_{2} \mathrm{D}}^{m}, \chi_{4}=$ $(1-\rho) \rho \eta \bar{\gamma}^{2}$ and $\nu_{4}=\eta \bar{\gamma} \rho d_{\mathrm{SR}_{2}}^{m} \kappa$.

The system outage probability for HD using AF is calculated using (36), where $F_{\gamma_{\mathrm{e} 2 e, \mathrm{R}_{1}}^{\mathrm{AF}}}(\kappa)$ and $F_{\gamma_{\mathrm{e} 2 \mathrm{e}, \mathrm{R}_{2}}^{\mathrm{AF}}}(\kappa)$ are shown in (61) and (62), $F_{\gamma_{\mathrm{e} 2 \mathrm{e}, \mathrm{R}_{1}}^{\mathrm{AF}}}\left(\gamma_{\mathrm{th}}\right)$ and $F_{\gamma_{\mathrm{e} 2 \mathrm{e}, \mathrm{R}_{2}}^{\mathrm{AF}}\left(\gamma_{\mathrm{th}}\right) \text { can }}$ be calculated by replacing $\kappa$ in (61) and (62) with $\gamma_{\mathrm{th}}$.

\section{Numerical results}

In this section, we present numerical simulation results to verify our analytical expressions derived in the previous section. Unless otherwise stated, we set the source 
transmission rate as $R_{c}=3 \mathrm{bps} / \mathrm{Hz}$; hence, the outage threshold is expressed as $\gamma_{\mathrm{th}}=2^{R_{c}}-1=7$. The distances $d_{\mathrm{SR}_{1}}, d_{\mathrm{R}_{1} \mathrm{D}}, d_{\mathrm{SR}_{2}}$, and $d_{\mathrm{R}_{2} \mathrm{D}}$ are normalized to unit value. The path loss exponent is set to be $m=3$, while the energy conversion efficiency is set to be $\eta=0.4$. The power splitting ratio $\rho$ is set to be $\rho=0.6$, which is the optimal value in [4]. We also set $\lambda_{h_{\mathrm{SR}_{1}}}=1, \lambda_{h_{\mathrm{R}_{1} \mathrm{D}}}=1, \lambda_{f_{1}}=0.1, \lambda_{h_{\mathrm{SR}_{2}}}=$ $1, \lambda_{h_{R_{2} \mathrm{D}}}=1, \lambda_{f_{2}}=0.1$.

In Fig. 4, we investigate the impact of preselected threshold $\kappa$ on the performance of DSSC full-duplex system in terms of outage probability when the relay uses $\mathrm{AF}$ protocol. It is obvious that our simulation and analytical results are well matched, confirming the correctness of our analysis. The switching threshold $\kappa$ is an important parameter for the performance of DSSC which determines which branch is active in each transmission session. It can be observed from Fig. 4 that the system outage probability decreases significantly when the preselected threshold $\kappa$ increases and obtains the lowest value when $\kappa=\gamma_{\text {th }}=$ 7 , which verifies our formulation in Section 3. This is because the rise in $\kappa$ makes the branch switching more likely occur so that the performance of DSSC improves, which in turn, decreases the outage probability. Besides, we see that the optimal outage probability achieves when $\kappa=\gamma_{\text {th }}=7$ which represents the optimal case of DSSC that a system selects the best link between $R_{1}$ and $R_{2}$ links for each transmission session, which is shown as (37). However, when swiching threshold $\kappa$ continues to increase, the system outage probability also goes up. This makes sense since if the switching threshold is chosen as too high, the source is continuously switching between two relays, which results in poor outage performance.

Figure 5 presents the change in outage probability of DSSC full-duplex systems in terms of predesigned threshold $\kappa$ when the DF protocol is used at the selected relay.

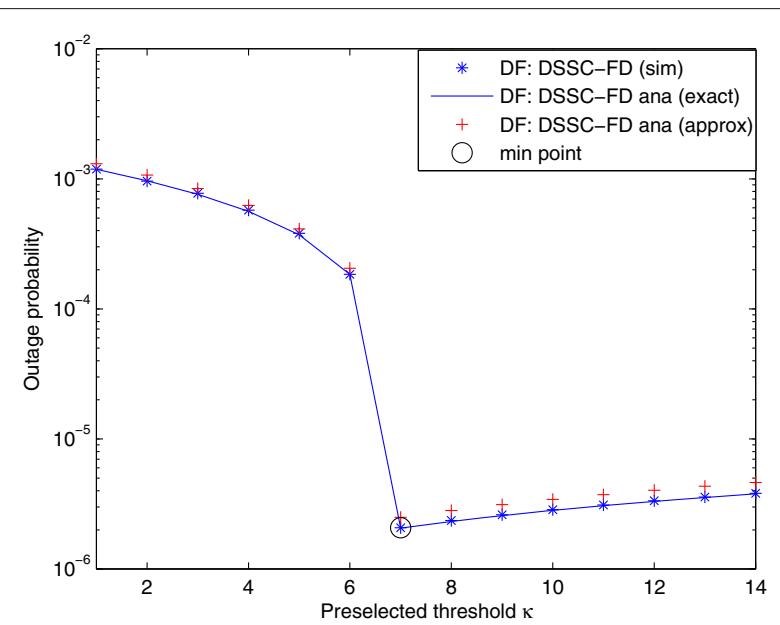

Fig. 5 Outage probability of DSSC full-duplex system with DF protocol versus $\kappa$ when $\gamma_{\text {th }}=7, \bar{\gamma}=50 \mathrm{~dB}$
Similar to the case of AF, the system outage probability decreases significantly when the predesigned threshold $\kappa$ increases and obtains the lowest value when $\kappa=\gamma_{\text {th }}=7$, which verifies our formulation in Section 3. This makes sense as the $\kappa$ increases, the branch switching rate happens more often and thus the outage performance is improved. Especially, when $\kappa=\gamma_{\text {th }}=7$, we obtain the best outage performance since DSSC system functions as a system which choose the best of $R_{1}$ and $R_{2}$ links for each transmission slot, which is presented in (29). However, the system outage probability increases when $\kappa$ continues to go up since the source has to switch continuously between two relays, which in turn, causes a negative effect on the outage performance. We can see that the exact analytical expression result, which is derived in (67), perfectly matched with simulation result and the tight approximation curve, which is expressed in (25), nearly matched with simulation result, thus verifies the correctness of our analysis.

In Fig. 6, we examine the outage probability between DSSC full-duplex and the conventional full-duplex systems with $\gamma_{\text {th }}=7, \kappa=3,7,11$ when the relay uses AF protocol. It can be shown from Fig. 6 that the simulation results are matched with the analytical results, confirming the correctness of our analysis. As can be readily observed, the outage probability of DSSC full-duplex system is much smaller than that of the conventional full duplex, confirming the positive effect of distributed switch and stay combining (DSSC) technique in improving the performance of the full-duplex relaying system. When the transmit SNR increases, the outage probability of both systems decreases. This is intuitive since when the transmit SNR increases, it means that the destination can

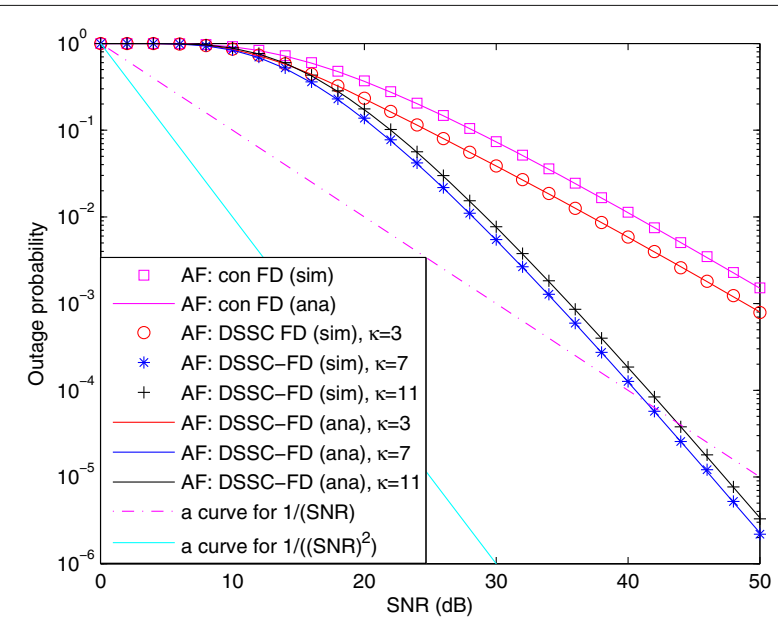

Fig. 6 Outage probability comparison between DSSC full-duplex system and the conventional full-duplex system with AF protocol when $\gamma_{\text {th }}=7, \kappa=3,7,11$ 
receive the better quality of the source information signal from the selected relay and the destination can decode the source signal more correctly, hence reduces the outage event at the destination. Besides that, once again we can also see that the outage probability of DSSC full-duplex system is minimum when $\gamma_{\text {th }}=\kappa=7$. Further, the diversity order achieved by our schemes is examined and compared. Recalling the diversity order can be indicated by the slope at high SNR regions of an outage performance curve [33]. This figure shows that the performance curves of two cases $\gamma_{\text {th }}<\kappa$ (corresponds to $\kappa=11$ ) and $\gamma_{\text {th }}=\kappa$ (corresponds to $\kappa=7$ ) are parallel to the curve that is proportional to $\frac{1}{\mathrm{SNR}^{2}}$, which provide the full diversity order while the performance curves of the case $\gamma_{\text {th }}>\kappa$ (corresponds to $\kappa=3$ ) and the conventional full duplex are parallel to the curve that is proportional to $\frac{1}{\mathrm{SNR}}$, which results in the diversity order of one. This makes sense when $\kappa \geq \gamma_{\text {th }}$ the system full diversity can be achieved with order of two since a large switching threshold makes the switch mechanism effective and the two branches can be efficiently exploited. In contrast, when $\kappa<\gamma_{\text {th }}$, the diversity order of system gradually goes down to one because a small $\kappa$ makes the branch switching less likely happen so that diversity can not be efficiently used.

Figure 7 illustrates the comparison between DSSC fullduplex and the conventional full-duplex system in terms of outage probability when $\gamma_{\mathrm{th}}=7, \kappa=3,7,11$ in case of using DF protocol at the relay. As can be seen from Fig. 7 , our simulation results are perfectly matched with the analytical expressions. From Fig. 7, the outage probability of DSSC full-duplex system is smaller than that of the conventional full-duplex one, which is the same as in the case of AF. The increase in transmit SNR also makes the

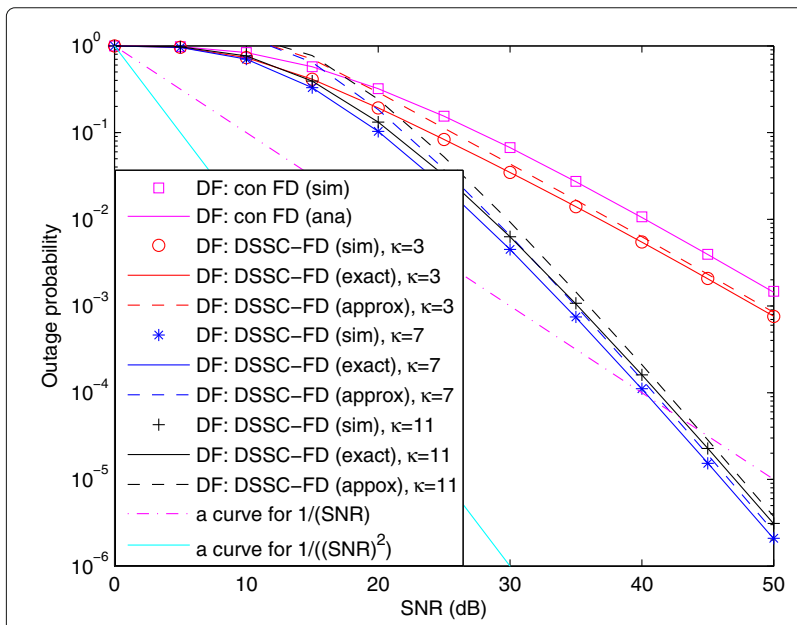

Fig. 7 Outage probability comparison between the DSSC full-duplex system and the conventional full-duplex system with DF protocol when $\gamma_{\mathrm{th}}=7, \kappa=3,7,11$ decrease in outage probability of both DSSC full-duplex and the conventional full-duplex systems which improves the system performance. The reason of this is the same as in the case of AF. Similar to the case of AF, this figure indicates that two cases $\gamma_{\text {th }}<\kappa$ (corresponds to $\kappa=11$ ) and $\gamma_{\text {th }}=\kappa$ (corresponds to $\kappa=7$ ) provide the full diversity order while the case $\gamma_{\text {th }}>\kappa$ (corresponds to $\kappa=3$ ) and the conventional full duplex have only diversity order of one. This verifies our correctness of diversity analysis which is conducted in Section 4.

Figure 8 compares the outage probability of DSSC full-duplex, DSSC half-duplex, and the conventional fullduplex systems for both AF and DF protocols when $\gamma_{\mathrm{th}}=$ $\kappa=7$. It can be shown from Fig. 8 that for all systems, the outage probability of DF protocol is slightly smaller than that of AF protocol. Besides that, when SNR increases, the performance of the DSSC full-duplex for both AF and DF protocol is significantly better than that of the DSSC halfduplex and conventional full-duplex system. This makes sense since with FD technique, the selected relay can simultaneously harvest energy from the source and forward the source information to destination, whereas with HD technique the selected relay has to separate energy harvesting process and information processing by using power splitting ratio $\rho$, which is clear advantage of FD compared with HD. Further, we can see that the performance of FD and HD can be improved significantly with the use of DSSC compared with the conventional FD system without DSSC.

Next, the impact of energy conversion efficiency $\eta$ on the performance of the DSSC full-duplex and the conventional full duplex with AF protocol is considered in Fig. 9. As shown in Fig. 9, the outage probability of both schemes goes down along with the increase of $\eta$. This is intuitive

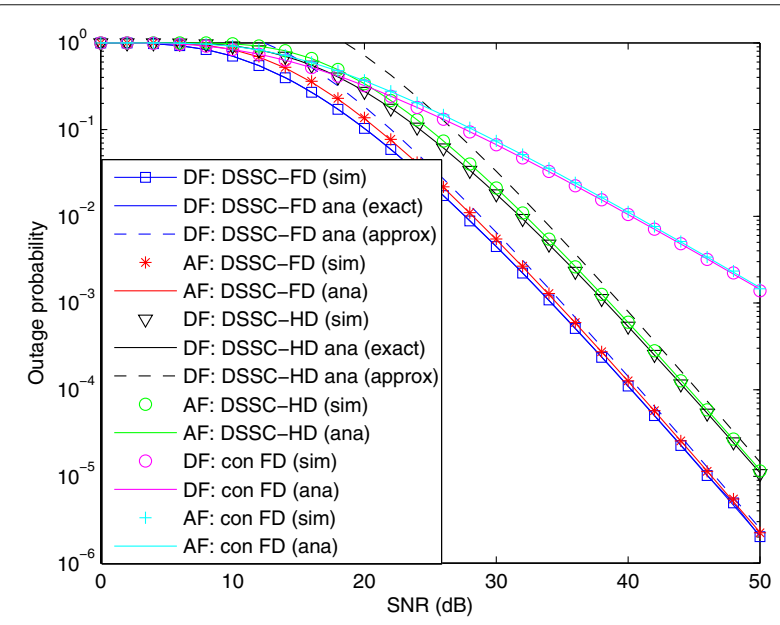

Fig. 8 Outage probability comparison between the DSSC full-duplex, DSSC half-duplex, and the conventional full-duplex system with DF and AF protocol when $\gamma_{\text {th }}=\kappa=7$ 


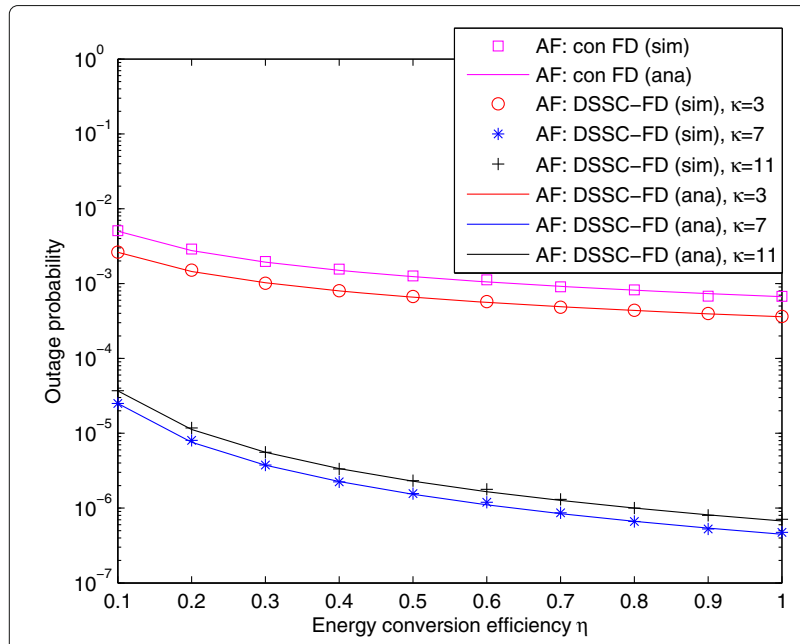

Fig. 9 Effects of energy conversion efficiency $\eta$ on outage probability of the DSSC full-duplex and the conventional full-duplex system with AF protocol when $\gamma_{\text {th }}=7, \kappa=3,7,11$

since when $\eta$ goes up, the amount of energy converted and used for the source information transmission at the selected relay also increases. It leads to the better received signal quality at the destination and thus improves the system performance. From Fig. 9, we also see that when $\eta$ increase, the performance of the DSSC full duplex is much better than that of the conventional full duplex in both cases $\gamma_{\text {th }}<\kappa$ (corresponds to $\kappa=11$ ) and $\gamma_{\text {th }}=\kappa$ (corresponds to $\kappa=7$ ).

Further, we also investigate the effect of energy conversion efficiency $\eta$ in the case of DF protocol for both the DSSC full-duplex and the conventional full-duplex system in Fig. 10. Similar to the case of DF, the increase in $\eta$ also improves the performance of both systems and in both cases $\gamma_{\text {th }}<\kappa$ (corresponds to $\kappa=11$ ) and $\gamma_{\text {th }}=\kappa$ (corresponds to $\kappa=7$ ) the performance of the DSSC fullduplex system is much better than that of the conventional full-duplex system.

\section{Conclusions}

In this paper, we investigated the performance of DSSC technique in dual-hop full-duplex relaying systems with wireless information and power transfer (WIPT). We obtained tight approximate expression and analytical expression of the system outage probability for DF and AF protocols over Rayleigh fading channels, respectively. Compared to the DSSC half-duplex relaying and conventional full-duplex relaying with WIPT system, our results show that DSSC technique can substantially boost the operational performance of full-duplex relaying systems and achieves the full spatial diversity in cases of the

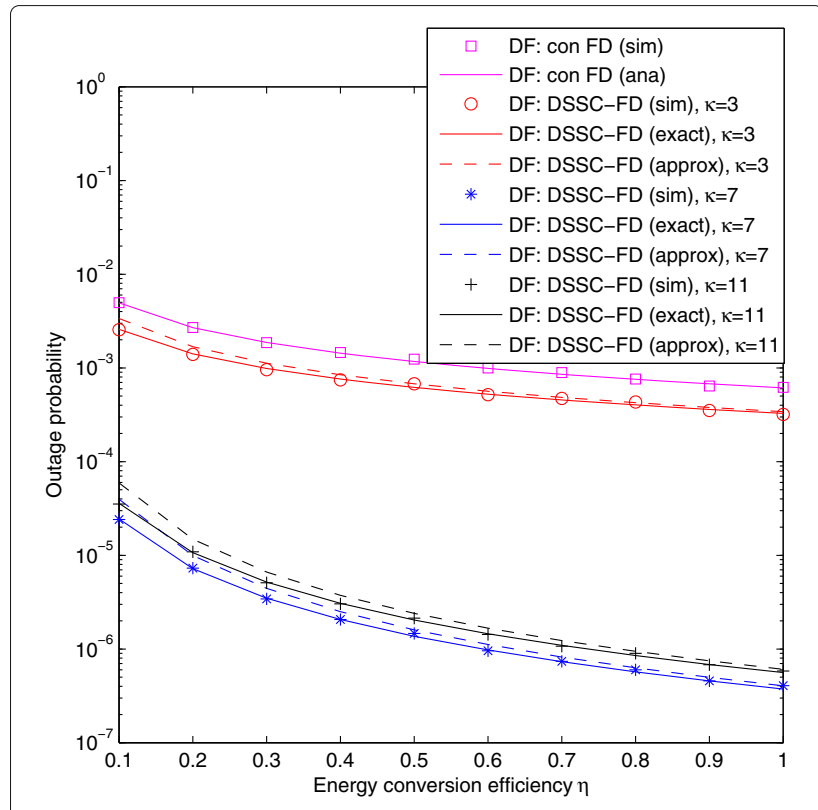

Fig. 10 Effects of energy conversion efficiency $\eta$ on outage probability of the DSSC full-duplex and the conventional full-duplex system with DF protocol when $\gamma_{\text {th }}=7, \kappa=3,7,11$

preselected switching threshold is chosen equal or higher than the outage threshold.

\section{Appendix}

\section{The proof of Lemma 1}

$F_{\gamma_{\mathrm{e} 2 \mathrm{e}, \mathrm{R}_{1}}^{\mathrm{DF}}}(\kappa)$ is expressed as follows

$$
\begin{aligned}
F_{\gamma_{\mathrm{e} 2, \mathrm{R}_{1}}^{\mathrm{DF}}}(\kappa) & =\operatorname{Pr}\left(\gamma_{\mathrm{e} 2 \mathrm{e}, \mathrm{R}_{1}}^{\mathrm{DF}}<\kappa\right) \\
& =\operatorname{Pr}\left(\min \left\{\gamma_{\mathrm{SR}_{1}}, \gamma_{\mathrm{R}_{1} \mathrm{D}}\right\}<\kappa\right) \\
& =\operatorname{Pr}\left(\min \left\{\frac{\left|h_{\mathrm{SR}_{1}}\right|^{2} \bar{\gamma}}{d_{\mathrm{SR}_{1}}^{m}}, \frac{\left|h_{\mathrm{R}_{1} \mathrm{D}}\right|^{2} \eta\left|h_{\mathrm{SR}_{1}}\right|^{2} \bar{\gamma}}{d_{\mathrm{R}_{1} \mathrm{D}}^{m}\left(d_{\mathrm{SR}_{1}}^{m}-\eta\left|f_{\mathrm{R}_{1}}\right|^{2}\right)}\right\}<\kappa\right) .
\end{aligned}
$$

Denoting $X=\left|h_{\mathrm{SR}_{1}}\right|^{2}, Y=\left|h_{\mathrm{R}_{1} \mathrm{D}}\right|^{2}$ and $U=\left|f_{\mathrm{R}_{1}}\right|^{2},(63)$ can be rewritten as

$$
\begin{aligned}
F_{\gamma_{\mathrm{e} 2 \mathrm{e}, \mathrm{R}_{1}}^{\mathrm{DF}}}(\kappa)= & \operatorname{Pr}\left(\min \left\{\frac{X \bar{\gamma}}{d_{\mathrm{SR}_{1}}^{m}}, \frac{Y \eta X \bar{\gamma}}{d_{\mathrm{R}_{1} \mathrm{D}}^{m}\left(d_{\mathrm{SR}_{1}}^{m}-\eta U\right)}\right\}<\kappa\right) \\
= & \int_{0}^{\infty} \operatorname{Pr}\left(\min \left\{\gamma_{\mathrm{SR}_{1} \mid X}, \gamma_{\mathrm{R}_{1} \mathrm{D} \mid X}\right\}<\kappa \mid X=x\right) \\
& \times f_{X}(x) d x
\end{aligned}
$$


where $\gamma_{\mathrm{SR}_{1} \mid X}=\frac{x \bar{\gamma}}{d_{\mathrm{SR}_{1}}^{m}}$ and $\gamma_{\mathrm{R}_{1} \mathrm{D} \mid X}=\frac{Y \eta x \bar{\gamma}}{d_{\mathrm{R}_{1} \mathrm{D}}^{m}\left(d_{\mathrm{SR}_{1}}^{m}-\eta U\right)}$. Since $\gamma_{\mathrm{SR}_{1} \mid X}$ and $\gamma_{\mathrm{R}_{1} \mathrm{D} \mid X}$ are independent, after some algebraic manipulations, we have

$$
\begin{aligned}
F_{\gamma_{\mathrm{e} 2 \mathrm{e}, \mathrm{R}_{1}}^{\mathrm{DF}}}(\kappa)= & \int_{0}^{\infty}\left[1-\operatorname{Pr}\left(\gamma_{\mathrm{SR}_{1} \mid X} \geq \kappa\right) \operatorname{Pr}\left(\gamma_{\mathrm{R}_{1} \mathrm{D} \mid X} \geq \kappa\right)\right] f_{X}(x) d x \\
= & \int_{0}^{\infty} f_{X}(x) d x-\int_{0}^{\infty} \operatorname{Pr}\left(\frac{x \bar{\gamma}}{d_{\mathrm{SR}_{1}}^{m}} \geq \kappa\right) \\
& \times \operatorname{Pr}\left(\frac{Y \eta x \bar{\gamma}}{d_{\mathrm{R}_{1} \mathrm{D}}^{m}\left(d_{\mathrm{SR}_{1}}^{m}-\eta U\right)} \geq \kappa\right) f_{X}(x) d x .
\end{aligned}
$$

Since $\int_{0}^{\infty} f_{X}(x) d x=1,(65)$ can be further expressed as

$$
\begin{aligned}
F_{\gamma_{\mathrm{e} 2 \mathrm{R}, \mathrm{R}_{1}}^{\mathrm{DF}}}(\kappa) & =1-\int_{\mu}^{\infty}\left[1-\operatorname{Pr}\left(Y<\frac{\kappa d_{\mathrm{R}_{1} \mathrm{D}}^{m}\left(d_{\mathrm{SR}_{1}}^{m}-\eta U\right)}{\eta x \bar{\gamma}}\right)\right] f_{X}(x) d x \\
& =1-\int_{\mu}^{\infty} f_{X}(x) d x+\int_{\mu}^{\infty} F_{Y}\left(\frac{\kappa d_{\mathrm{R}_{1} \mathrm{D}}^{m}\left(d_{\mathrm{SR}_{1}}^{m}-\eta U\right)}{\eta x \bar{\gamma}}\right) f_{X}(x) d x,
\end{aligned}
$$

where $\mu=\frac{\kappa d_{\mathrm{SR}_{1}}^{m}}{\bar{\gamma}}$. Since $X=\left|h_{\mathrm{SR}_{1}}\right|^{2}$ and $Y=\left|h_{\mathrm{R}_{1} \mathrm{D}}\right|^{2}$ follow exponential distributions with parameters $\frac{1}{\lambda_{h_{\mathrm{SR}_{1}}}}$ and $\frac{1}{\lambda_{h_{R_{1}} \mathrm{D}}}$, respectively, (66) can be expressed as

$$
\begin{aligned}
F_{\gamma_{\mathrm{e} 2 \mathrm{e}, \mathrm{R}_{1}}^{\mathrm{DF}}}(\kappa)= & -\int_{\mu}^{\infty} \frac{1}{\lambda_{h_{\mathrm{SR}_{1}}}} e^{-\frac{x}{\lambda_{\mathrm{SR}_{1}}}} d x \\
& +\int_{\mu}^{\infty}\left(1-e^{\left.-\frac{\kappa d_{\mathrm{R}_{1} \mathrm{D}}^{m}\left(d_{\mathrm{SR}_{1}}^{m}-\eta U\right)}{\eta x \bar{\gamma} h_{\mathrm{R}_{1} \mathrm{D}}}\right)} \frac{1}{\lambda_{h_{\mathrm{SR}_{1}}}} e^{-\frac{x}{\lambda_{\mathrm{SR}_{1}}}} d x\right. \\
= & 1-\frac{1}{\lambda_{h_{\mathrm{SR}_{1}}}} \underbrace{\int_{\mu}^{\infty} e^{-\frac{x}{\lambda_{\mathrm{SR}_{1}}}-\frac{\kappa d_{\mathrm{R}_{1} \mathrm{D}}^{m}\left(d_{\mathrm{SR}_{1}}^{m}-\eta U\right)}{\eta x \bar{\gamma} h_{\mathrm{R}_{1} \mathrm{D}}}} d x}_{\Phi} .
\end{aligned}
$$

Using Maclaurin's series for $e^{-\alpha / x}$ and with small $\mathrm{x}$, we have the approximation as $e^{-\alpha / x} \approx 1-\alpha / x, \Phi$ in (67) can be rewritten as

$$
\Phi \approx \int_{\mu}^{\infty} e^{-\frac{x}{\lambda h_{\mathrm{SR}_{1}}}} d x-\frac{\kappa d_{\mathrm{R}_{1} \mathrm{D}}^{m}\left(d_{\mathrm{SR}_{1}}^{m}-\eta U\right)}{\eta \bar{\gamma} \lambda_{h_{\mathrm{R}_{1} \mathrm{D}}}} \int_{\mu}^{\infty} \frac{e^{-\frac{x}{\lambda h_{\mathrm{SR}_{1}}}}}{x} d x
$$

With the help of ([34], Eq. (3.381.3)) and after some manipulation steps, the integral $\Phi$ can be solved as

$$
\begin{gathered}
\Phi \approx \lambda_{h_{\mathrm{SR}_{1}}} e^{-\frac{\mu}{\lambda_{\mathrm{SR}_{1}}}}-\frac{\kappa d_{\mathrm{R}_{1} \mathrm{D}}^{m}\left(d_{\mathrm{SR}_{1}}^{m}-\eta U\right)}{\eta \bar{\gamma} \lambda_{h_{\mathrm{R}_{1} \mathrm{D}}}} \Gamma\left(0, \frac{\mu}{\lambda_{h_{\mathrm{SR}_{1}}}}\right) \\
\approx \lambda_{h_{\mathrm{SR}_{1}}} e^{-\frac{\mu}{\lambda_{\mathrm{SR}_{1}}}}-\frac{\kappa d_{\mathrm{R}_{1} \mathrm{D}}^{m} d_{\mathrm{SR}_{1}}^{m}}{\eta \bar{\gamma} \lambda_{h_{\mathrm{R}_{1} \mathrm{D}}}} \Gamma\left(0, \frac{\mu}{\lambda_{h_{\mathrm{SR}_{1}}}}\right) \\
+\underbrace{\frac{\kappa d_{\mathrm{R}_{1} \mathrm{D}}^{m} U}{\bar{\gamma} \lambda_{h_{\mathrm{R}_{1} \mathrm{D}}}} \Gamma\left(0, \frac{\mu}{\lambda_{h_{\mathrm{SR}_{1}}}}\right)}_{\theta} .
\end{gathered}
$$

Now, conditioned on $\mathrm{U}, \theta$ in (69) can be shown as

$$
\begin{aligned}
\theta & =\int_{0}^{\infty} \frac{\kappa d_{\mathrm{R}_{1} \mathrm{D}}^{m} u}{\bar{\gamma} \lambda_{h_{\mathrm{R}_{1} \mathrm{D}}}} \Gamma\left(0, \frac{\mu}{\lambda_{h_{\mathrm{SR}_{1}}}}\right) \frac{1}{\lambda_{f_{\mathrm{R}_{1}}}} e^{-\frac{u}{\lambda_{\mathrm{R}_{1}}}} d u \\
& =\frac{\kappa d_{\mathrm{R}_{1} \mathrm{D}}^{m} \Gamma\left(0, \frac{\mu}{\lambda_{h_{\mathrm{S}_{1}}}}\right) \lambda_{f_{\mathrm{R}_{1}}}}{\bar{\gamma} \lambda_{h_{\mathrm{R}_{1} \mathrm{D}}}}
\end{aligned}
$$

where $\theta$ can be solved by using integration by parts and after some simple manipulations, we obtain the last result in (70).

Plugging (70) into (69) and then plugging (69) into (67), we obtain as follows

$$
\begin{aligned}
F_{\gamma_{\mathrm{e} 2, \mathrm{R}_{1}}^{\mathrm{DF}}}(\kappa) \approx & 1-e^{-\frac{\mu}{\lambda_{\mathrm{SR}_{1}}}}+\frac{\kappa d_{\mathrm{R}_{1} \mathrm{D}}^{m} d_{\mathrm{SR}_{1}}^{m}}{\eta \bar{\gamma} \lambda_{h_{\mathrm{R}_{1} \mathrm{D}}} \lambda_{h_{\mathrm{SR}_{1}}}} \Gamma\left(0, \frac{\mu}{\lambda_{h_{\mathrm{SR}_{1}}}}\right) \\
& -\frac{\kappa d_{\mathrm{R}_{1} \mathrm{D}}^{m} \Gamma\left(0, \frac{\mu}{\lambda_{h_{\mathrm{R}_{1}}}}\right) \lambda_{\mathrm{R}_{1}}}{\bar{\gamma} \lambda_{h_{\mathrm{R}_{1} \mathrm{D}}} \lambda_{h_{\mathrm{SR}_{1}}}} \\
\approx & 1-e^{-\frac{\mu}{\lambda_{\mathrm{SR}_{1}}}}+\frac{\kappa d_{\mathrm{R}_{1} \mathrm{D}}^{m} \Gamma\left(0, \frac{\mu}{\lambda_{\mathrm{SR}_{1}}}\right)\left(d_{\mathrm{SR}_{1}}^{m}-\eta \lambda_{f_{\mathrm{R}_{1}}}\right)}{\eta \bar{\gamma} \lambda_{h_{\mathrm{R}_{1} \mathrm{D}}} \lambda_{h_{\mathrm{SR}_{1}}}} .
\end{aligned}
$$

Finally, plugging $\mu=\frac{\kappa d_{\mathrm{SR}_{1}}^{m}}{\bar{\gamma}}$ into (71), we obtain the desired result as in (25).

\section{The proof of Lemma 2}

First, we denote $a=\kappa \bar{\gamma} d_{\mathrm{R}_{1} \mathrm{D}}^{m}\left(d_{\mathrm{SR}_{1}}^{m}-\eta\left|f_{\mathrm{R}_{1}}\right|^{2}\right), b=$ $\kappa d_{\mathrm{SR}_{1}}^{m} d_{\mathrm{R}_{1} \mathrm{D}}^{m}\left(d_{\mathrm{SR}_{1}}^{m}-\eta\left|f_{\mathrm{R}_{1}}\right|^{2}\right), c=\eta \bar{\gamma}^{2}$ and $d=\kappa \eta \bar{\gamma} d_{\mathrm{SR}_{1}}^{m}$. (33) can be rewritten as

$$
\begin{aligned}
F_{\gamma_{\mathrm{e} 2 e, \mathrm{R}_{1}}^{\mathrm{AF}}}(\kappa) & =\operatorname{Pr}\left(c\left|h_{\mathrm{SR}_{1}}\right|^{4}\left|h_{\mathrm{R}_{1} \mathrm{D}}\right|^{2}<a\left|h_{\mathrm{SR}_{1}}\right|^{2}+d\left|h_{\mathrm{SR}_{1}}\right|^{2}\left|h_{\mathrm{R}_{1} \mathrm{D}}\right|^{2}+b\right) \\
& \left.=\operatorname{Pr}\left(\left(c\left|h_{\mathrm{SR}_{1}}\right|^{4}-d\left|h_{\mathrm{SR}_{1}}\right|^{2}\right)\left|h_{\mathrm{R}_{1} \mathrm{D}}\right|^{2}<a\left|h_{\mathrm{SR}_{1}}\right|^{2}+b\right)\right)
\end{aligned}
$$


Then, (72) can be derived as

$F_{\gamma_{\mathrm{ee} e_{1} R_{1}}^{\mathrm{AF}}}(\kappa)= \begin{cases}\operatorname{Pr}\left(\left|h_{\mathrm{R}_{1} \mathrm{D}}\right|^{2}<\frac{a\left|h_{\mathrm{SR}_{1}}\right|^{2}+b}{c\left|h_{\mathrm{SR}_{1}}\right|^{4}-d\left|h_{\mathrm{SR}_{1}}\right|^{2}}\right), & \left|h_{\mathrm{SR}_{1}}\right|^{2}>d / c \\ \operatorname{Pr}\left(\left|h_{\mathrm{R}_{1} \mathrm{D}}\right|^{2}>\frac{a\left|h_{\mathrm{SR}_{1}}\right|^{2}+b}{c\left|h_{\mathrm{SR}_{1}}\right|^{4}-d\left|h_{\mathrm{SR}_{1}}\right|^{2}}\right)=1, & \left|h_{\mathrm{SR}_{1}}\right|^{2}<d / c\end{cases}$

where the second equality in (73) is true since if $\left|h_{\mathrm{SR}_{1}}\right|^{2}<$ $d / c, c\left|h_{\mathrm{SR}_{1}}\right|^{4}-d\left|h_{\mathrm{SR}_{1}}\right|^{2}$ will be a negative number and the probability of $\left|h_{R_{1} \mathrm{D}}\right|^{2}$ is always greater than negative number. Therefore, the probability of $\left|h_{R_{1} D}\right|^{2}$ is always 1 . Next, denoting $X=\left|h_{\mathrm{SR}_{1}}\right|^{2}$, (73) is further given by

$$
\begin{aligned}
F_{\gamma_{\mathrm{e} 2 \mathrm{R}, \mathrm{R}_{1}}^{\mathrm{AF}}}(\kappa)= & \int_{0}^{d / c} \operatorname{Pr}\left(\left|h_{\mathrm{R}_{1} \mathrm{D}}\right|^{2}>\frac{a x+b}{c x^{2}-d x}\right) f_{X}(x) d x \\
& +\int_{d / c}^{\infty} \operatorname{Pr}\left(\left|h_{\mathrm{R}_{1} \mathrm{D}}\right|^{2}<\frac{a x+b}{c x^{2}-d x}\right) f_{X}(x) d x \\
= & \int_{0}^{d / c} f_{X}(x) d x+\int_{d / c}^{\infty}\left(1-e^{-\frac{a x+b}{\left(c x^{2}-d x\right) \lambda_{\mathrm{R}_{1} \mathrm{D}} \mathrm{D}}}\right) f_{X}(x) d x \\
= & 1-\frac{1}{\lambda_{h_{\mathrm{SR}}}} \int_{d / c}^{\infty} e^{-\left(\frac{a x+b}{\left(c x^{2}-d x\right) \lambda_{\mathrm{R}_{1} \mathrm{D}}}+\frac{x}{\lambda_{h_{\mathrm{SR}}}}\right)} d x,
\end{aligned}
$$

where $\int_{0}^{d / c} f_{X}(x) d x+\int_{d / c}^{\infty} f_{X}(x) d x=\int_{0}^{\infty} f_{X}(x) d x=1$ and $f_{X}(x)=\frac{1}{\lambda_{h_{\mathrm{SR}_{1}}}} e^{-\frac{x}{\lambda_{\mathrm{SR}_{1}}}}$, the last equality in (74) can be achieved.

Plugging $a=\kappa \bar{\gamma} d_{\mathrm{R}_{1} \mathrm{D}}^{m}\left(d_{\mathrm{SR}_{1}}^{m}-\eta\left|f_{\mathrm{R}_{1}}\right|^{2}\right), b=$ $\kappa d_{\mathrm{SR}_{1}}^{m} d_{\mathrm{R}_{1} \mathrm{D}}^{m}\left(d_{\mathrm{SR}_{1}}^{m}-\eta\left|f_{\mathrm{R}_{1}}\right|^{2}\right), c=\eta \bar{\gamma}^{2}$ and $d=\kappa \eta \bar{\gamma} d_{\mathrm{SR}_{1}}^{m}$ into (74), (74) can be rewritten as

$$
\begin{aligned}
& F_{\gamma_{\mathrm{e} 2 e, \mathrm{R}_{1}}^{\mathrm{AF}}}(\kappa)=1-\frac{1}{\lambda_{h_{\mathrm{SR}_{1}}}} \\
& \times \int_{\frac{\kappa d_{\mathrm{SR}_{1}}^{m}}{\bar{\gamma}}}^{\infty} e^{-\frac{\kappa d_{\mathrm{R}_{1} \mathrm{D}}^{m}\left(d_{\mathrm{SR}_{1}}^{m}-\eta \mid \mathrm{R}_{1} L^{2}\right)\left(\bar{\gamma} x+d_{\mathrm{SR}_{1}}^{m}\right)}{\left.\left(\eta \bar{\gamma}^{2} x^{2}-\kappa \eta \bar{\gamma} d_{\mathrm{SR}_{1}}^{m}\right)^{2}\right)_{h_{\mathrm{R}_{1} \mathrm{D}} \mathrm{D}}}-\frac{x}{\lambda_{\mathrm{SR}_{1}}}} d x .
\end{aligned}
$$

The integration in (75) cannot be further simplified. At high SNRs, we can apply a approximation, so $d_{\mathrm{SR}_{1}}^{m}$ can be neglected. Thus, (75) can be expressed as

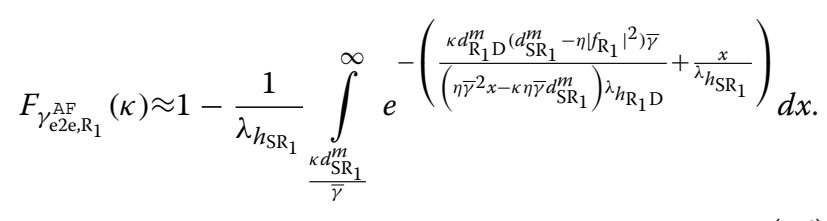

Let we define $v=\eta \bar{\gamma}^{2} x-\kappa \eta \bar{\gamma} d_{\mathrm{SR}_{1}}^{m}$. Thus, (76) can be rewritten as

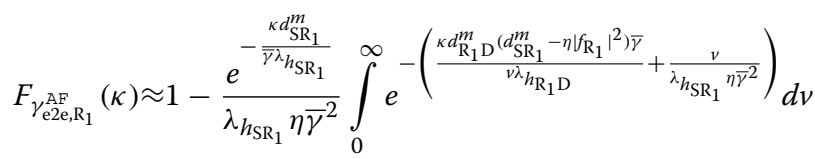

$$
\begin{aligned}
& \approx 1-e^{-\frac{\kappa d_{\mathrm{SR}_{1}}^{m}}{\bar{\gamma} h_{\mathrm{SR}_{1}}}} \sqrt{\frac{4 \kappa d_{\mathrm{R}_{1} \mathrm{D}}^{m}\left(d_{\mathrm{SR}_{1}}^{m}-\eta\left|f_{\mathrm{R}_{1}}\right|^{2}\right)}{\lambda_{h_{\mathrm{SR}_{1}}} \eta \bar{\gamma} \lambda_{h_{\mathrm{R}_{1} \mathrm{D}}}}} \\
& \times K_{1}\left(\sqrt{\frac{4 \kappa d_{\mathrm{R}_{1} \mathrm{D}}^{m}\left(d_{\mathrm{SR}_{1}}^{m}-\eta\left|f_{\mathrm{R}_{1}}\right|^{2}\right)}{\lambda_{h_{\mathrm{SR}_{1}}} \eta \bar{\gamma} \lambda_{h_{\mathrm{R}_{1} \mathrm{D}}}}}\right),
\end{aligned}
$$

where $K_{1}($.$) is the first-order modified Bessel function of$ the second kind ([34], Eq. (8.432.1)) and the last equality is obtained by using the formula in ([34], Eq. (3.324.1)).

Next, denoting $U=\left|f_{\mathrm{R}_{1}}\right|^{2}$ and conditioned on $U$ from (77), we obtain the desired result as in (34).

\section{Acknowledgments}

The work of Q. N. Le, N. T. Do and B. An was supported by Basic Science Research Program through the National Research Foundation of Korea (NRF) funded by the Ministry of Education (Grant No. 2016R1D1A1B03934898) and by the Leading Human Resource Training Program of Regional Neo industry Through the National Research Foundation of Korea (NRF) funded by the Ministry of science, ICT and future planning (Grant No. 2016H1D5A1910577). The work of V. N. Q. Bao was funded by Vietnam National Foundation for Science and Technology Development (NAFOSTED) under Grant No. 102.04-2014.32.

\section{Competing interests}

The authors declare that they have no competing interests.

\section{Author details}

${ }^{1}$ Department of Electronics and Computer Engineering, Hongik University, Sejong 30016, Korea. ${ }^{2}$ Department of Wireless Communications, Posts and Telecommunications Institute of Technology, Ho Chi Minh City 710372 , Vietnam. ${ }^{3}$ Department of Computer and Information Communications Engineering, Hongik University, Sejong 30016, Korea.

Received: 28 June 2016 Accepted: 28 November 2016

Published online: 15 December 2016

\section{References}

1. W Lumpkins, Nikola Tesla's dream realized: wireless power energy harvesting. IEEE Consum, Electron. Mag. 3(1), 39-42 (2014)

2. RL Varshney, in Proc. of the IEEE International Symposium on Information Theory (ISIT 2008). Transporting information and energy simultaneously, (Toronto, ON, Canada, 2008), pp. 1612-1616

3. P Grover, A Sahai, in Proc. of the IEEE International Symposium on Information Theory (ISIT 2010). Shannon meets Tesla: wireless information and power transfer, (Austin, 2010), pp. 2363-2367

4. AA Nasir, X Zhou, S Durrani, R Kennedy, Relaying protocols for wireless energy harvesting and information processing. IEEE Trans. Wireless Commun. 12(7), 3622-3636 (2013) 
5. AA Nasir, X Zhou, S Durrani, AR Kennedy, in Proc. IEEE ICC. Throughput and ergodic capacity of wireless energy harvesting based DF relaying network, (Sydney, 2014), pp. 4066-4071

6. AA Nasir, X Zhou, S Durrani, AR Kennedy, Wireless-powered relays in cooperative communications: time-switching relaying protocols and throughput analysis. IEEE Trans. Commun. 63(5), 1607-1622 (2015)

7. AH Suraweera, I Krikidis, G Zheng, C Yuen, JP Smith, Low complexity end-to-end performance optimization in MIMO full-duplex relay systems. IEEE Trans. Wireless Commun. 13(2), 913-927 (2014)

8. PB Day, RA Margetts, WD Bliss, P Schniter, Full-duplex MIMO relaying: achievable rates under limited dynamic range. IEEE J. Sel. Areas Commun. 30(8), 1541-1553 (2012)

9. I Krikidis, AH Suraweera, JP Smith, C Yuen, Full-duplex relay selection for amplify-and-forward cooperative networks. IEEE Trans. Wireless Commun. 11(12), 4381-4393 (2012)

10. A Sabharwal, et al., In-band full-duplex wireless: challenges and opportunities. IEEE J. Sel. Areas Commun. 32(9), 1637-1652 (2014)

11. I Krikidis, G Zheng, B Ottersten, in IEEE 23rd International Symposium on Wireless Communications and Networking Conference (WCNC). Harvest-use cooperative networks with half/full-duplex relaying, (Shanghai, China, 2013), pp. 4256-4260

12. C Zhong, H Suraweera, G Zheng, I Krikidis, Z Zhang, Wireless information and power transfer with full duplex relaying. IEEE Trans. Commun. 62(10), 3447-3461 (2014)

13. M Mohammadi, KB Chalise, AH Suraweera, C Zhong, G Zheng, I Krikidis, Throughput analysis and optimization of wireless-powered multiple antenna full-duplex relay systems. IEEE Trans. Commun. 64(4), 1769-1785 (2016)

14. H Ju, R Zhang, Optimal resource allocation in full-duplex wireless powered communication network. IEEE Trans. Commun. 62(10), 3528-3540 (2014)

15. Y Zeng, R Zhang, Full-duplex wireless-powered relay with self energy recycling. IEEE Wirel. Commun. Lett. 4(2), 201-204 (2015)

16. HC Yang, MS Alouini, Performance analysis of multibranch switched diversity systems. IEEE Trans. Commun. 51(5), 782-794 (2003)

17. MS Alouini, HC Yang, Minimum estimation and combining generalized selection combining (MEC-GSC). IEEE Trans. Wireless Commun. 6(2), 526-532 (2007)

18. A Gharanjik, K Mohamed-pour, Switch-and-stay partial relay selection over Rayleigh fading channels. IET Commun. 5(9), 1199-1203 (2011)

19. M Yan, Q Chen, X Lei, QT Duong, P Fan, Outage probability of switch and stay combining in two-way amplify-and-forward relay networks. IEEE Wirel. Commun. Lett. 1(4), 296-299 (2012)

20. KM Jataprolu, SD Michalopoulos, R Schober, Colocated and distributed switch-and-stay combining: optimality under switching rate constraints. IEEE Trans. Veh. Technol. 63(1), 451-457 (2014)

21. DS Michalopoulos, GK Karagiannidis, Distributed switch and stay combining (DSSC) with a single decode and forward relay. IEEE Commun. Lett. 11(5), 408-410 (2007)

22. DS Michalopoulos, GK Karagiannidis, Two-relay distributed switch and stay combining. IEEE Trans. Commun. 56(11), 1790-1794 (2008)

23. H Suraweera, D Michalopoulos, G Karagiannidis, Performance of distributed diversity systems with a single amplify-and-forward relay. IEEE Trans. Vehicular Tech. 58(5), 2603-2608 (2009)

24. VNQ Bao, HY Kong, Distributed switch and stay combining for selection relay networks. IEEE Commun. Lett. 13(12), 914-916 (2009)

25. T Tran-Thien, T Do-Hong, VNQ Bao, in Third International Conference on Communications and Electronics (ICCE). Outage probability of selection relaying networks with distributed switch and stay combining over Rayleigh fading channels (Nha Trang, Viet Nam, 2010), pp. 61-64

26. Z Paruk, $\mathrm{HXu}$, Distributed switch and stay combining with partial relay selection and signal space diversity. IET Commun. 8(1), 105-113 (2014)

27. T Riihonen, S Werner, R Wichman, Hybrid full-duplex/half-duplex relaying with transmit power adaptation. IEEE Trans. Wireless Commun. 10(9), 3074-3085 (2011)

28. C Tellambura, A Annamalai, VK Bhargava, Unified analysis of switched diversity systems in independent and correlated fading channels. IEEE Trans. Commun. 49, 1955-1965 (2001)

29. KH Liu, Performance analysis of relay selection for cooperative relays based on wireless power transfer with finite energy storage. IEEE Trans. Veh. Technol. 65(7), 5110-5121 (2016)
30. L Zheng, DNC Tse, Diversity and multiplexing: a fundamental tradeoff in multiple-antenna channels. IEEE Trans. Inf. Theory. 49(5), 1073-1096 (2003)

31. Z Ding, SM Perlaza, I Esnaola, HV Poor, Power allocation strategies in energy harvesting wireless cooperative networks. IEEE Trans. Wirel. Commun. 13(2), 846-860 (2014)

32. G Zhu, C Zhong, HA Suraweera, GK Karagiannidis, Z Zhang, TA Tsiftsis, Wireless information and power transfer in relay systems with multiple antennas and interference. IEEE Trans. Commun. 63(4), 1400-1418 (2015)

33. Z Ding, HV Poor, Multi-user SWIPT cooperative networks: is the max-min criterion still diversity-optimal? IEEE Trans.Wirel. Commun. 15(1), 553-567 (2016)

34. IS Gradshteyn, IM Ryzhik, Table of integrals, series, and products. (Elsevier, New York, 2007)

\section{Submit your manuscript to a SpringerOpen ${ }^{\circ}$ journal and benefit from:}

- Convenient online submission

- Rigorous peer review

- Immediate publication on acceptance

- Open access: articles freely available online

- High visibility within the field

- Retaining the copyright to your article

Submit your next manuscript at $>$ springeropen.com 